\title{
Orphan penumbrae: Submerging horizontal fields ${ }^{\star}$
}

\author{
J. Jurčák ${ }^{1}$, L. R. Bellot Rubio ${ }^{2}$, and M. Sobotka ${ }^{1}$ \\ 1 Astronomical Institute of the Academy of Sciences, Fričova 298, 25165 Ondřejov, Czech Republic \\ e-mail: jurcak@asu.cas.cz \\ ${ }^{2}$ Instituto de Astrofísica de Andalucía (CSIC), Apdo. Correos 3004, 18080 Granada, Spain
}

Received 22 July 2013 / Accepted 6 February 2014

\begin{abstract}
Aims. We investigate the properties of orphan penumbrae, which are photospheric filamentary structures observed in active regions near polarity inversion lines that resemble the penumbra of regular sunspots but are not connected to any umbra.

Methods. We use Hinode data from the Solar Optical Telescope to determine the properties of orphan penumbrae. Spectropolarimetric data are employed to obtain the vector magnetic field and line-of-sight velocities in the photosphere. Magnetograms are used to study the overall evolution of these structures, and $G$-band and $\mathrm{Ca}$ II $\mathrm{H}$ filtergrams are to investigate their brightness and apparent horizontal motions.

Results. Orphan penumbrae form between regions of opposite polarity in places with horizontal magnetic fields. Their magnetic configuration is that of $\Omega$-shaped flux ropes. In the two cases studied here, the opposite-polarity regions approach each other with time and the whole structure submerges as the penumbral filaments disappear. Orphan penumbrae are very similar to regular penumbrae, including the existence of strong gas flows. Therefore, they could have a similar origin. The main difference between them is the absence of a "background" magnetic field in orphan penumbrae. This could explain most of the observed differences.

Conclusions. The fast flows we detect in orphan penumbrae may be caused by the siphon flow mechanism. Based on the similarities between orphan and regular penumbrae, we propose that the Evershed flow is also a manifestation of siphon flows.
\end{abstract}

Key words. Sun: magnetic fields - Sun: photosphere - sunspots

\section{Introduction}

All structures seen in the solar photosphere other than granulation are caused by magnetic fields that influence the convection and thus the presence and shape of granules. The largest, strongest, and most complex magnetic fields are located in active regions. The fields can completely inhibit convective motions there, and thus create sunspots and pores (see Keppens \& Martinez Pillet 1996; Solanki 2003; Schlichenmaier 2009; Borrero \& Ichimoto 2011). There are other structures observed in active regions that are associated with the submergence or emergence of magnetic flux tubes but not so thoroughly studied as the more prominent sunspots and pores.

In this paper, we analyse filamentary structures observed in active regions near polarity inversion lines. Since they visually resemble a penumbra without an umbra, we call them "orphan penumbrae" following Zirin \& Wang (1991). Similar structures were described before. There are numerous reports of alignments of granules or intergranular lanes near polarity inversion lines of active regions (first by Miller 1960). Loughhead \& Bray (1961) interpreted the alignments as being due to magnetic loops rising through the solar photosphere. The same conclusion was reached by other authors, although the reported lifetimes and sizes of the structures differ considerably (Brants \& Steenbeek 1985; Tarbell et al. 1990; Wang \& Zirin 1992; Schlichenmaier et al. 2010). In a recent work, Kuckein et al. (2012) suggested that orphan penumbrae are the photospheric

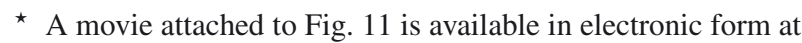
http: //www . aanda.org signatures of low-lying flux ropes. However, as pointed out by Lim et al. (2013) and Zuccarello et al. (2014), other mechanisms can also lead to the formation of orphan penumbrae. These authors observed orphan penumbrae in regions with overlaying chromospheric fields and interpreted them as a consequence of rising $\Omega$-loops that are trapped in the photosphere by the latter. Here, we suggest that the $\Omega$-loops responsible for orphan penumbrae submerge again during their decay phase.

The presence of inclined magnetic fields in the photosphere may indeed explain the formation of orphan penumbrae from at least a theoretical perspective. Chandrasekhar (1961) showed that convection in inclined magnetic fields is modulated by the strength of the vertical field component, while the horizontal component determines the shape of the convective cells. This was observationally supported by Jurčák (2011), assuming that penumbral filaments are of convective origin. Numerical simulations also confirm the importance of the horizontal component of the magnetic field for the convective cell shape (see Thomas \& Weiss 2008). If the magnetic field is horizontal, convection will not be stopped but the cells will be highly elongated and resemble filaments, which perhaps, explains the existence of orphan penumbrae.

Here, we determine the physical properties of orphan penumbrae during their decay phase using spectropolarimetric measurements, magnetograms, and $G$-band and Ca II H filtergrams taken by the Hinode satellite. Section 2 describes the observations and data analysis. The magnetic and dynamic configuration of orphan penumbrae is inferred and compared with that of regular penumbrae in Sect. 3. The evolution, brightness, 


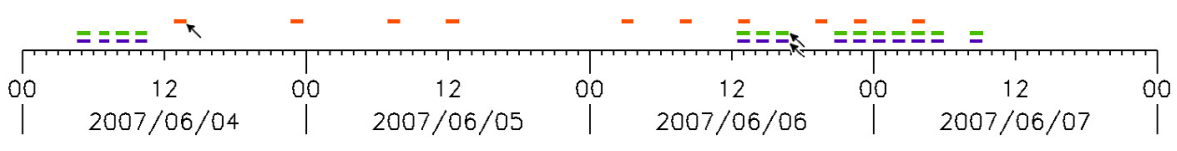

\begin{tabular}{|c|c|c|c|c|c|}
\hline & & & & 7 & \\
\hline 0 & $\begin{array}{c}12 \\
2010 / 07 / 22\end{array}$ & 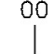 & $\begin{array}{c}12 \\
2010 / 07 / 23\end{array}$ & oo & $\begin{array}{c}12 \\
2010 / 07 / 24\end{array}$ \\
\hline
\end{tabular}

Fig. 1. Chart showing the observations of active region NOAA 10960. The blue and green dashes indicate BFI observations in $G$-band and $\mathrm{Ca}$ II $\mathrm{H}$, respectively. The red dashes indicate spectropolarimetric scans. The arrows mark the data shown and discussed in this paper.

Fig. 2. Analogous to Fig. 1 but for active region NOAA 11089. The yellow colour indicates NFI $V / I$ magnetograms in the NaI D. All the NFI $V / I$ magnetograms are used in this paper, so they are not marked individually by arrows. and horizontal motions of these structures are also discussed there. We interpret the various observational results in Sect. 4 and summarise our conclusions in Sect. 5.

\section{Observations and data analysis}

We use data obtained with the three instruments of the Solar Optical Telescope (SOT, Tsuneta et al. 2008) aboard the Hinode satellite (Kosugi et al. 2007), namely, the Broad-band Filter Imager (BFI), the Narrow-band Filter Imager (NFI), and the Spectropolarimeter (SP). The BFI data were taken through a $G$-band filter centred at $430.5 \mathrm{~nm}$ with a bandpass of $0.8 \mathrm{~nm}$ and through a Ca II H filter centred at $396.85 \mathrm{~nm}$ with a bandpass of $0.3 \mathrm{~nm}$. The pixel sampling corresponds to $0{ }^{\prime} 11(2 \times$ 2 binning), which results in a diffraction-limited spatial resolution of 0.22 . The NFI measurements were acquired in the red wing of the $\mathrm{NaI} \mathrm{D}$ line with the polarisation unit to obtain $V / I$ magnetograms at a spatial resolution of 0.32 (pixel sampling of 0.'16). The SP observed the Stokes profiles of the two Fe I lines at 630.15 and $630.25 \mathrm{~nm}$. The SP measurements were taken in the so-called fast mode, which is when the pixel sampling is 0.32 , the exposure time is $1.6 \mathrm{~s}$, and the resulting noise level is $10^{-3} I_{\mathrm{c}}$. The data were calibrated with the standard routines available in the Hinode SolarSoft package. A subset of these observations has been analysed by Zuccarello et al. (2014).

According to Shelyag et al. (2004), the $G$-band forms at around $\tau_{500}=-1$, which is comparable to the formation height of $100 \mathrm{~km}$ reported by Jess et al. (2012). Since the typical granulation pattern is observed in the $G$-band images, the formation height has to be below $140 \mathrm{~km}$, which is the boundary between normal and reverse granulation (Cheung et al. 2007). Because of the width of the Hinode $\mathrm{Ca}$ II $\mathrm{H}$ filter and the broad response/contribution functions of the line (as computed for the FALC model by Carlsson et al. 2007 or Rezaei et al. 2008), the formation height of the Ca II H images is not well defined. They mostly sample photospheric layers below $300 \mathrm{~km}$ (reverse granulation pattern in quiet Sun regions), but there are also contributions from the chromosphere. The Hinode Ca II $\mathrm{H}$ filtergrams show strong emission at the position of enhanced magnetic activity. The Na I D magnetograms were taken +14 pm from the line core. This part of the line wing is formed around $200 \mathrm{~km}$ above the solar surface (Leenaarts et al. 2010). According to Cabrera Solana et al. (2005), the two iron lines recorded by the SP are most sensitive to layers around $\tau_{500}=-1$. The physical parameters obtained from the inversion of the Fe I lines, therefore, give us information about this atmospheric height.

The observations used here correspond to two active regions (NOAA 10960 and 11089) and are summarised in Figs. 1 and 2. In both cases, we did not capture the formation of the orphan penumbrae. We observed their evolution and decaying phase only. The data marked with arrows in Figs. 1 and 2 are presented in detail in this paper. However, all the measurements were analysed to study the evolution of the orphan penumbrae and to confirm the validity of our results.

In the case of NOAA 10960 (Fig. 1), there are unevenly separated SP scans of the active region, and the orphan penumbra is located on the southern edge of the field of view (FOV). There is a two-day gap in the BFI data as the structure was out of the FOV of this instrument. In the initial sequence of BFI images taken on 4 June 2007, the cadence of the filtergrams is $60 \mathrm{~s}$ for both datasets, while it is $100 \mathrm{~s}$ for the $G$-band images and $20 \mathrm{~s}$ for the $\mathrm{Ca}$ II $\mathrm{H}$ filtergrams in the rest of the BFI data. The active region was located between $\left(47^{\circ} \mathrm{E}, 6^{\circ} \mathrm{S}\right)$ and $\left(6^{\circ} \mathrm{E}, 6^{\circ} \mathrm{S}\right)$ during the analysed time period (heliocentric angles between $48^{\circ}$ and $6^{\circ}$ ).

We have at our disposal SP scans of active region NOAA 11089 that repeat approximately every $100 \mathrm{~min}$ (Fig. 2). Moreover, there are almost uninterrupted observations of Na I D V/I magnetograms with a cadence of 5 min. However, the photospheric evolution of NOAA 11089 is not well covered, since only $13 \mathrm{BFI}$ images were taken through the $G$-band filter. This active region moved from $\left(31^{\circ} \mathrm{E}, 27^{\circ} \mathrm{S}\right]$ to $\left[14^{\circ} \mathrm{E}, 28^{\circ} \mathrm{S}\right)$ during the observations (heliocentric angles between $43^{\circ}$ and $32^{\circ}$ ).

\subsection{Data analysis}

We used the SIR inversion code (Stokes Inversion based on Response functions; Ruiz Cobo \& del Toro Iniesta 1992) to retrieve the values of the atmospheric parameters from the Stokes profiles observed by the Hinode SP. The pixel sampling of $0 . ' 32$ is comparable to the width of the studied filaments, so they are not spatially resolved. For this exploratory analysis, we adopted a simple model atmosphere assuming that the physical conditions do not change with optical depth with the exception of temperature. (We allowed for three optical depths at which the temperature can change and obtained the stratification in a finer grid by interpolation.) We took into account the spectral point spread function of the Hinode SP, allowed for micro-turbulent velocity, and set the macro-turbulent velocity to zero. We did not use any stray light profile.

The magnetic field inclinations and azimuths retrieved from the inversion are expressed in the line-of-sight (LOS) reference frame. We applied the code AMBIG (Leka et al. 2009) to solve the $180^{\circ}$ ambiguity of the LOS azimuth. The AMBIG code is based on the minimum energy method (Metcalf 1994). The disambiguated LOS vector magnetic field was then transformed to the local reference frame (LRF) with the help of routines from the AZAM code (Lites et al. 1995).

We used local correlation tracking (LCT, November \& Simon 1988) to study horizontal motions in the $G$-band and Ca II H filtergrams. Applied to a time series of images, the LCT technique provides the time-averaged horizontal velocity field of all structures in the FOV. We first aligned the BFI images and 

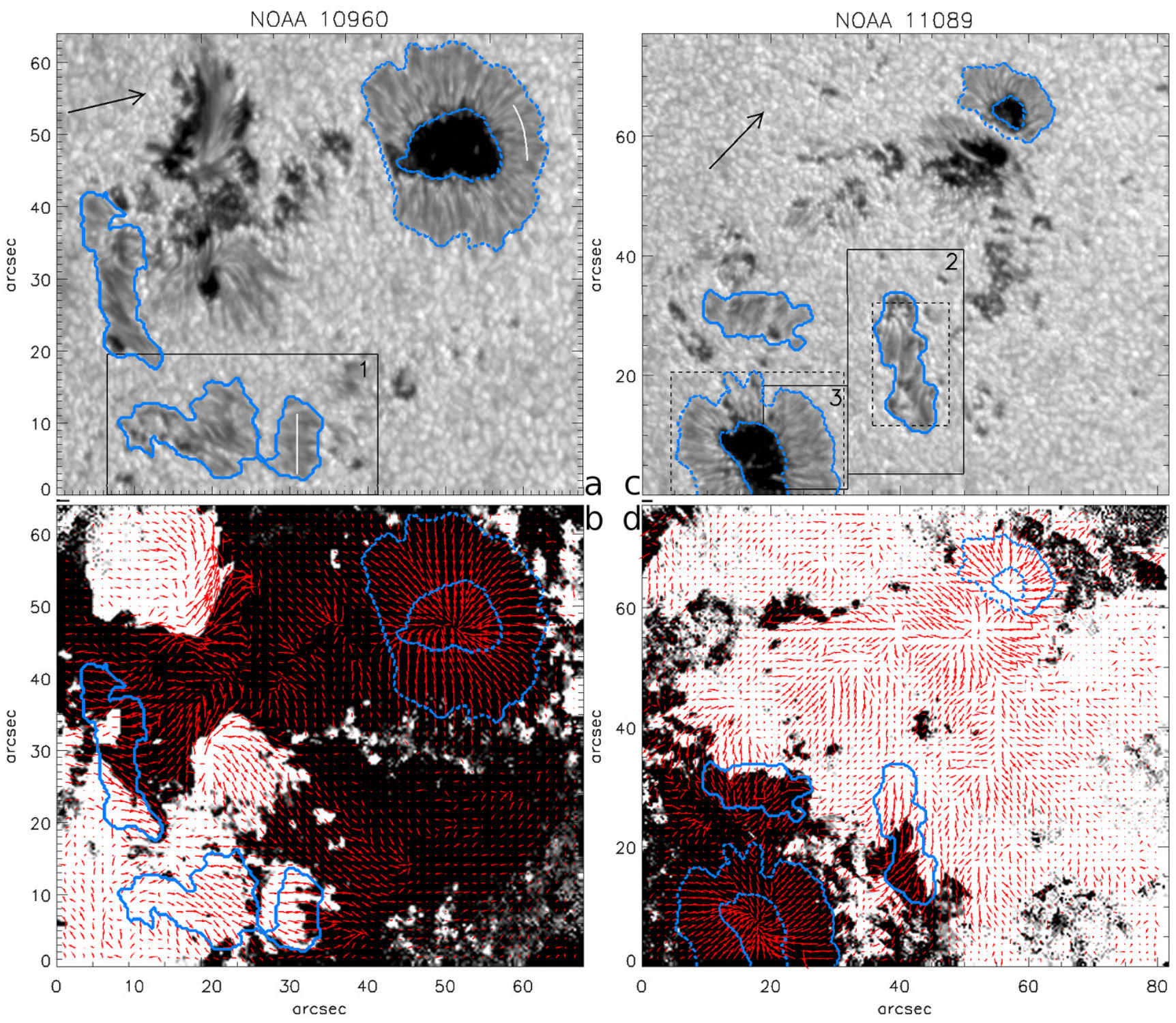

Fig. 3. Continuum intensity maps showing an active region NOAA 10960 on 4 June 2007 around 13 UT a) and NOAA 11089 on 22 July 2010 around 22:30 UT c) at heliocentric angles of $47^{\circ}$ and $43^{\circ}$, respectively. North is up and east to the left. Maps of the vertical component of the magnetic field in the LRF saturated at $\pm 25 \mathrm{G}$ are shown in b) and d). The red arrows mark the strength and orientation of the horizontal component of the magnetic field. The length of the thick lines above the maps b) and d) correspond to $1000 \mathrm{G}$. The solid and dashed contours encircle the orphan and regular penumbrae, respectively. The boxes indicated by solid black lines mark the areas displayed in Figs. 4 and 5. The boxes indicated by dashed black lines mark the areas displayed in Fig. 8. The white lines in a) mark the positions of cuts displayed in Fig. 6.

removed the p-mode oscillations by applying a $k-\omega$ filter with a cut-off of $5 \mathrm{~km} \mathrm{~s}^{-1}$. Then, we selected a Gaussian tracking window of FWHM $1^{\prime \prime}$ (9 pixels), which is fine enough to obtain proper motions of structures within filamentary regions, and the temporal integration was made over an interval of $18 \mathrm{~min}$ for both $G$-band and $\mathrm{Ca}$ II $\mathrm{H}$ data. The resulting velocity maps do not represent real gas flows; for example, there are motions of structures towards the umbra in most penumbrae, although the gas flow is in the opposite direction (Sobotka et al. 1999).

\section{Results}

\subsection{Magnetic and velocity structure}

In Fig. 3, we present continuum intensity maps of active regions NOAA 10960 and 11089 constructed from the SP scans with solid and dashed contours encircling orphan and regular penumbrae. To show the location of the orphan penumbrae with respect to the polarity inversion line (PIL), we created highly saturated maps of the vertical component of the magnetic field ( $\pm 25 \mathrm{G}$; Figs. 3b,d) using the magnetic field strengths and LRF inclinations derived from the inversion.

In all cases, the orphan penumbral segments are formed between regions of opposite polarity and, indeed, most of them lie on PILs. When orphan penumbrae sit in unipolar areas, the opposite polarities are found in pore-like structures located at the end of some of the filaments. In Figs. $3 \mathrm{~b}$ and $3 \mathrm{~d}$, the orientation and strength of the horizontal component of the vector magnetic field in the LFR is marked by red arrows. As can be seen, the orphan penumbral filaments and the magnetic field are aligned and cross the PILs from the positive to the negative side (normal configuration). The filaments of the orphan penumbrae observed by Kuckein et al. (2012) were found to be aligned with the magnetic field too. However, they ran parallel to the PIL instead of 


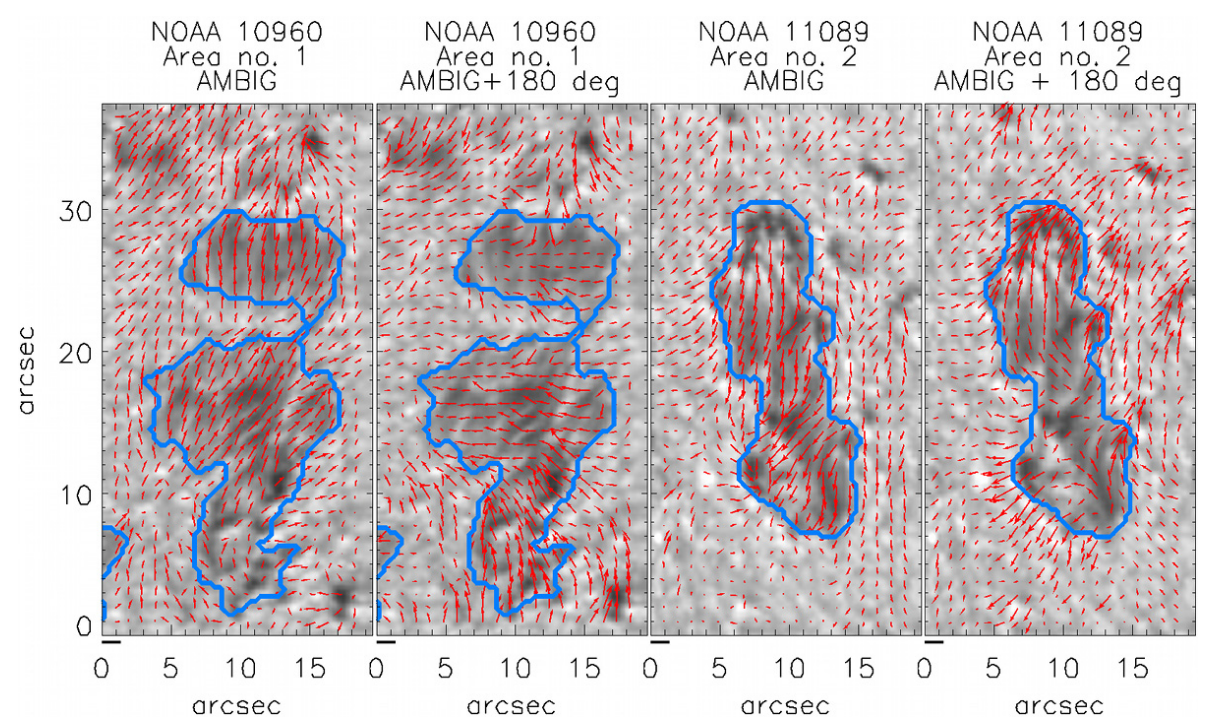

Fig. 4. Continuum intensity maps showing the orphan penumbrae in areas No. 1 and 2 of Fig. 3. The arrows represent the orientation and strength of the horizontal component of the vector magnetic field in the LRF. For each of the areas, we display the two LRF azimuths that result from the two possible LOS azimuth solutions. The left panels show the correct solution. The lengths of the thick lines near the beginning of the $x$ axes correspond to $1000 \mathrm{G}$. Area No. 1 is rotated by $90^{\circ}$ for display purposes. crossing it, which was ascribed to the existence of a chromospheric filament above them.

In Fig. 4, we show the two possible orientations of the LRF azimuth for the orphan penumbrae of areas No. 1 and 2 in Fig. 3. The solution displayed on the left panels corresponds to that determined by the AMBIG code. We believe this is the correct solution, because it leads to a magnetic field well aligned with the penumbral filaments. Such a solution implies an $\Omega$-shaped configuration of the magnetic field lines. The solution on the right panels corresponds to the opposite orientation of the LOS azimuth and produces a LRF magnetic field that is not aligned with the filaments. This scenario does not seem realistic, and therefore, we discard it. All the orphan penumbrae observed in active regions NOAA 10960 and 11089 are compatible with $\Omega$-shaped flux ropes.

Figure 5 displays maps of continuum intensity, magnetic field inclination in the LRF $\left(\gamma_{\mathrm{LRF}}\right)$, magnetic field strength $(B)$, and LOS velocity ( $\left.v_{\mathrm{LOS}}\right)$ as derived from the inversion of the two areas. For comparison, we also display a segment of a regular penumbra using the same colour coding.

From the LRF inclination maps of Fig. 5, it is clear that the orphan penumbral filaments form in regions where the field is mostly horizontal and that they stretch across the PIL, confirming our interpretation of the saturated $B_{z}$ maps of Fig. 3. Their magnetic field inclination is within $\pm 10^{\circ}$ from being completely horizontal. Near the footpoints of the filaments, the field is inclined by $30^{\circ}$ with respect to the local normal line. In the centreside part of the regular penumbra (area No. 3), the LRF inclination map shows that the field turns horizontal radially outwards while keeping the polarity of the spot (as described by numerous authors, see the review by Solanki 2003).

The magnetic field strength is around $900 \mathrm{G}$ in orphan penumbrae with generally weaker fields in area No. 1 compared to area No. 2. Near the footpoints of the filaments, the field tends to be stronger. A detailed analysis of the variation of the magnetic field strength along orphan penumbral filaments is presented in Sect. 4. In the regular penumbra, we observe a steady decrease of the field strength from the umbra towards the outer sunspot edge (Solanki 2003).

The LOS velocity maps displayed in Fig. 5 make it clear that orphan and regular penumbrae have a similar flow structure. The flows are aligned with the filaments and become faster further from the location of their origin, as it also happens in sunspots (see Borrero et al. 2005). The velocity peaks are always reached at the filament ends, which allows us to determine the tails of the filaments. In the case of redshifted flows (away from the observer), this behaviour can to some extend be caused by projection effects as the flow turns towards the solar surface and has a more favourable orientation to the LOS. However, the flow gets faster further from the location of their origin also in the case of flows oriented towards the observer (blueshifted segments), where the angle between the flow direction and the LOS is larger (less favourable orientation to the LOS).

A unique property of orphan penumbrae is the existence of flows of opposite direction in neighboring filaments. Regular penumbrae do not normally show such oppositely-directed motions, although there are cases of penumbral segments with inward gas flows during the initial phases of their evolution in the Hinode data archive. As described later, the oppositely oriented flows observed in orphan penumbrae eventually disappear.

In Fig. 6, we compare the variation of parameters along cuts perpendicular to filaments in orphan and regular penumbrae (see white lines in Fig. 3a). Since orphan and regular penumbrae were observed at the same heliocentric angle and analysed with the same inversion technique, the magnetic and dynamic parameters we obtain should be fully comparable.

In the orphan penumbra, there are small variations of the LRF inclination across the filaments with maximum changes of $10^{\circ}$. In the regular penumbra, bright and dark filaments show larger differences of up to $20^{\circ}$. The field strength differences between bright and dark filaments are also larger in the regular penumbra (more than $100 \mathrm{G}$ ) compared to the orphan penumbra, where there are global changes of $B$, but no pronounced fluctuations across individual filaments. Likewise, variations of the LOS velocity are more prominent across regular penumbral filaments $\left(2 \mathrm{~km} \mathrm{~s}^{-1}\right)$ than across orphan penumbral filaments (up to $\left.1 \mathrm{~km} \mathrm{~s}^{-1}\right)$.

We do not observe significant correlations between continuum intensity and magnetic or dynamic parameters along the cuts in either orphan or regular penumbrae. However, there is a clear tendency of faster flows in weaker and more horizontal magnetic fields in the regular penumbra, which can be interpreted as being due to the presence of a steady, stronger, and more vertical background magnetic field. Along the orphan penumbral cut, the faster flows tend to be located in regions with stronger and more horizontal fields. This is not compatible with 
J. Jurčák et al.: Orphan penumbrae: Submerging horizontal fields
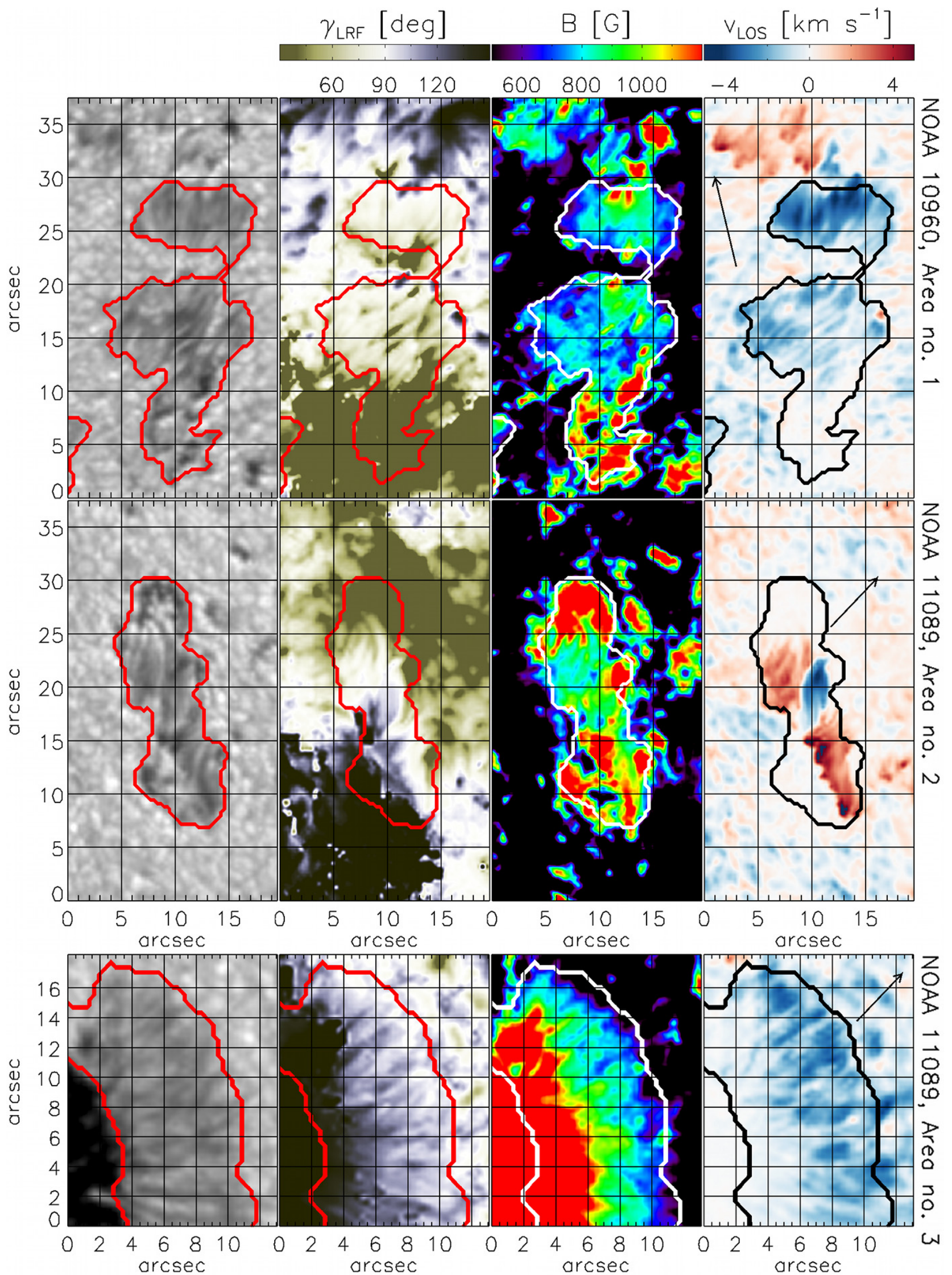

Fig. 5. Left to right: maps of continuum intensity, LRF magnetic field inclination $\left(\gamma_{\mathrm{LRF}}\right)$, magnetic field strength $(B)$, and LOS velocity $\left(v_{\mathrm{LOS}}\right)$ in the areas marked by solid black boxes in Fig. 3. Area No. 1 is rotated by $90^{\circ}$ for display purposes. The contours are the same as in Fig. 3. Areas No. 1 and No. 2 show the properties of orphan penumbrae segments and area No. 3 of the centre side of regular penumbra. The arrows in the LOS velocity maps point towards the disc centre. 


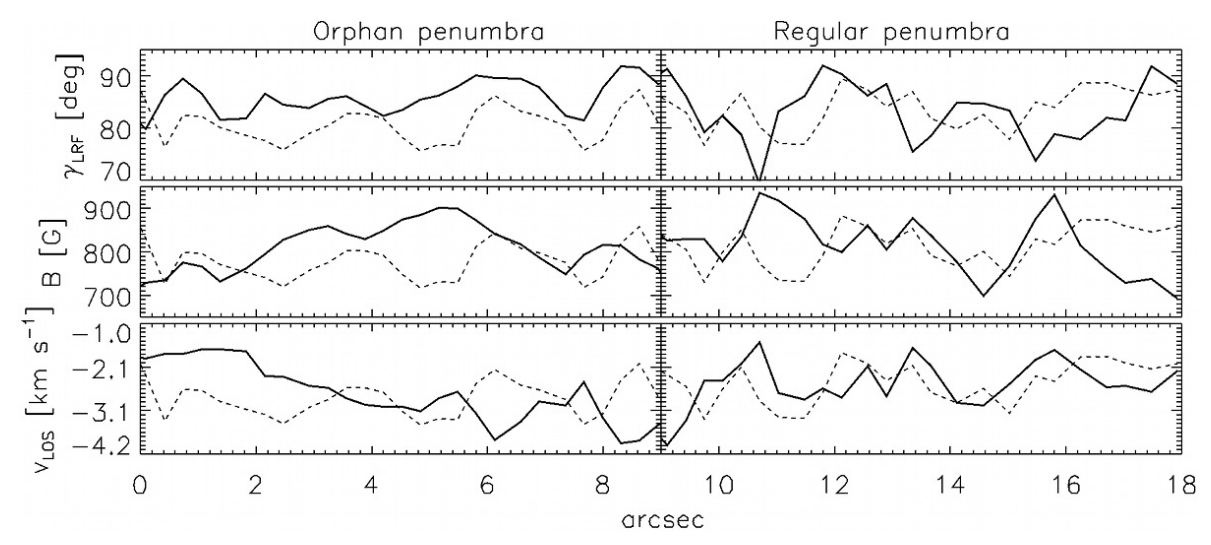

Fig. 6. Variations of $\gamma_{\mathrm{LRF}}, B$, and $v_{\mathrm{LOS}}$ along the cuts marked with white lines in Fig. 3. The solid and dashed lines show the atmospheric parameters and the continuum intensity, respectively. For display purposes, we changed the polarity of the field along the cut through the filaments of the regular penumbra and also increased $\gamma_{\mathrm{LRF}}$ by $10^{\circ}$ and $v_{\mathrm{LOS}}$ by $1 \mathrm{~km} \mathrm{~s}^{-1}$ there. The range of continuum intensities used to draw the dashed lines is the same for orphan and regular penumbrae.

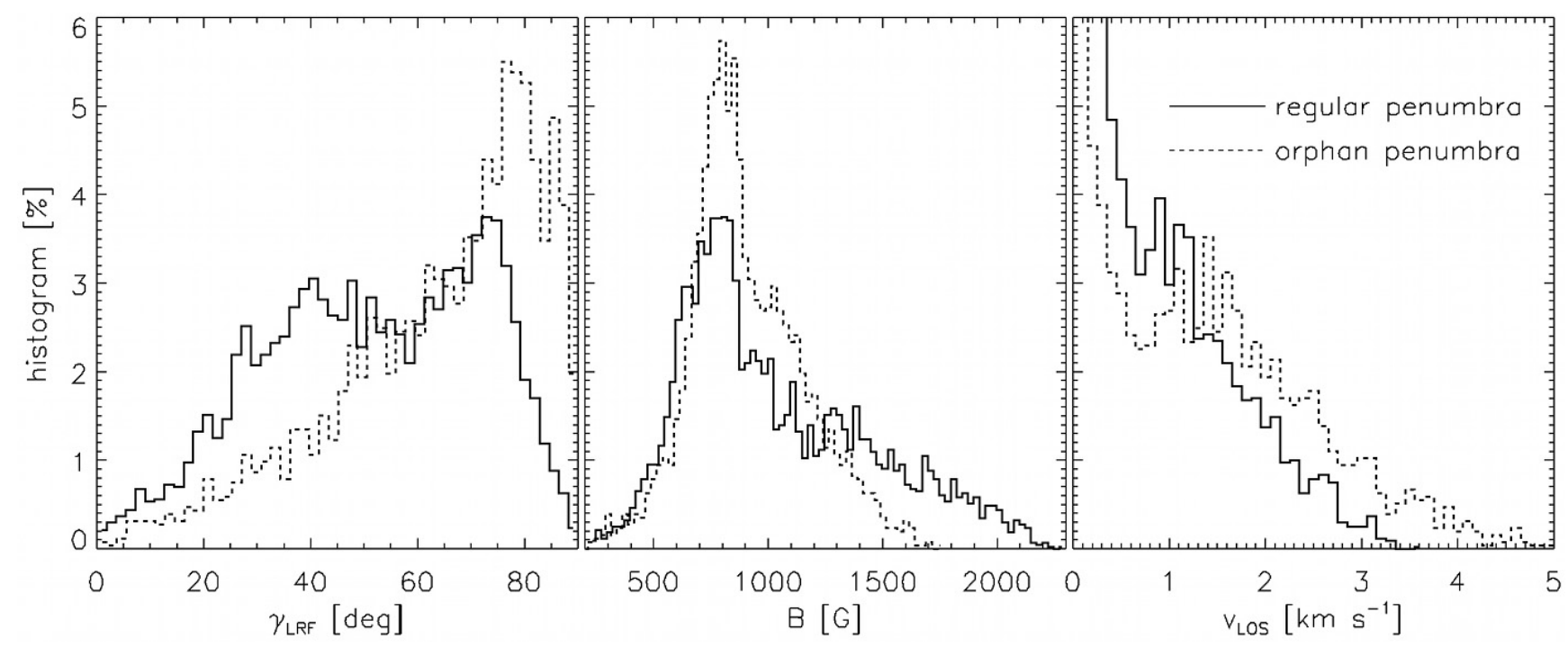

Fig. 7. Histograms of the LRF magnetic field inclination (left; $90^{\circ}$ is horizontal), magnetic field strength (middle), and absolute values of the LOS velocity (right) in regular and orphan penumbrae. The pixels used to create the regular penumbra histograms correspond to those in the two regular penumbrae marked by dashed lines in Fig. 3c. The orphan penumbra histograms are based on the pixels in two orphan penumbrae encircled by solid contours in Fig. 3c.

the existence of a background magnetic field. A detailed analysis of the relation between flow velocity and field strength in orphan penumbrae is carried out in Sect. 4.

To compare the global properties of orphan and regular penumbrae, we present histograms of $\gamma_{\mathrm{LRF}}, B$, and $v_{\mathrm{LOS}}$ in Fig. 7. This analysis is based on the segments of orphan and regular penumbrae marked with solid and dashed contours in Fig. 3c.

The left panel of Fig. 7 shows the field inclination distributions in orphan and regular penumbrae. As mentioned before, the magnetic field is mostly horizontal in orphan penumbrae, whereas regular penumbrae show inclinations ranging from $25^{\circ}$ to $80^{\circ}$. This result can be understood by keeping in mind that the information about the magnetic field inclination (and strength) in regular penumbrae is dominated by the sunspot background field, and our simplified inversion scheme cannot account for the field component harboring the gas flow (whose inclination increases with radial distance and even submerges in the midpenumbra and beyond, see the review by Borrero \& Ichimoto 2011). Pixels with more vertical magnetic fields are located outside of the filamentary regions of orphan penumbrae.

In the middle panel of Fig. 7, we present histograms of the field strengths in orphan and regular penumbrae. Similarly to the field inclination, there is a significantly larger spread of $B$ in regular penumbrae compared to orphan penumbrae. This is again likely caused by the behaviour of the background magnetic field in regular penumbrae, whose strength steadily decreases from the umbra to the outer sunspot edge. Pixels with $B$ over $1200 \mathrm{G}$ are located outside of the filamentary parts of orphan penumbrae.

The right panel of Fig. 7 shows the LOS velocity distributions of both types of penumbrae. Unlike the magnetic field strength and inclination, the shapes of the distributions are comparable. This could be due to the fact that the background magnetic field component does not contain fast flows (Borrero et al. 2004; Bellot Rubio et al. 2004) and thus does not significantly influence the retrieved values of the LOS velocity, which is almost exclusively determined by the flow component. Pixels with LOS velocities smaller than $0.5 \mathrm{~km} \mathrm{~s}^{-1}$ are located near the umbra/penumbra boundary in regular penumbrae and in pore-like structures near the ends of orphan penumbrae filaments.

The only difference in the flow field of orphan and regular penumbrae is the amplitude of the LOS velocity. We found maximum LOS velocities of $5 \mathrm{~km} \mathrm{~s}^{-1}$ and $3.7 \mathrm{~km} \mathrm{~s}^{-1}$, respectively. This is to some extent due to projection effects, because we observe only blueshifted regions on the centre side of regular penumbrae (like in area No. 3). As mentioned before, the orientation of the flow with respect to the LOS is more favourable in redshifted regions than in blueshifted regions near the filaments tails, where the flow is fastest. However, the flows observed in the blueshifted regions of the orphan penumbrae are also faster than those detected in the regular penumbra. This may point to a 


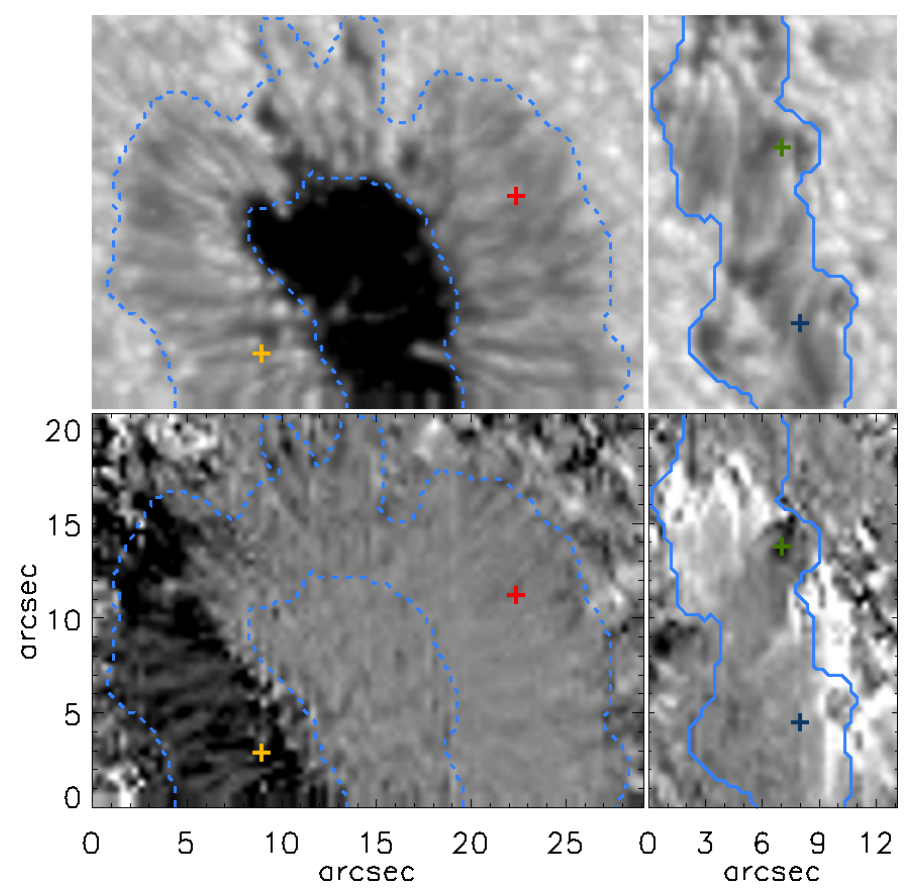

Fig. 8. Continuum intensities (top) and Stokes $V$ area asymmetries (bottom) within the dashed black boxes of Fig. 3c. The area asymmetry maps are saturated at \pm 0.5 . The coloured + symbols mark the positions of the Stokes profiles shown in Fig. 9.

different submergence angle of the filaments in the two types of structures.

\subsection{Stokes profile asymmetries}

Although they are not common, highly asymmetric Stokes profiles can be observed in orphan penumbrae. This is demonstrated by the maps of Stokes $V$ area asymmetry $\left(\sum_{\lambda} V / \sum_{\lambda}|V|\right)$ of Fig. 8.

Very asymmetric Stokes $V$ profiles tend to occur at the border of the filamentary region of the orphan penumbra, while there are only small asymmetries elsewhere. The centre side of the regular penumbra also shows mild asymmetries, whereas the limb side exhibits stronger asymmetries, although the magnetic configuration is the same on both penumbral sides. In Fig. 9, we present examples of observed Stokes profiles. In orphan and regular penumbrae, one can find highly asymmetric Stokes $V$ profiles (second and fourth rows, respectively) but also nearly symmetric profiles (first and third rows).

Zuccarello et al. (2014) interpreted the Stokes $V$ asymmetries of orphan penumbrae as a proof of the existence of a background magnetic field. However, this is not the only mechanism that can produce asymmetric Stokes profiles. Strong asymmetries are observed in canopy-like regions, where strong vertical gradients of the physical parameters occur (Ishikawa et al. 2010; Viticchié 2012; Sainz Dalda et al. 2012). The edges of orphan penumbrae show the largest asymmetries and are the places, where the configurations described by Ishikawa et al. (2010) and Viticchié (2012) may be more relevant (albeit of much larger size), given that the horizontal field has to bend rapidly to become more vertical and stronger at the footpoints of the $\Omega$-shaped loop that forms the penumbra. These are the places where the line of sight encounters the strongest vertical gradients, which would produce asymmetric Stokes profiles without the intervention of any background magnetic field. To estimate more precisely the configuration of magnetic field in these regions, we would have to employ a depth-dependent inversion of the observed Stokes profiles. This is beyond the scope of this paper.

Thus, we conclude that the absence of large Stokes asymmetries in the central part of orphan penumbrae is compatible with the absence of a background magnetic field in those structures.

\subsection{Time evolution}

Figure 10 shows the temporal evolution of the orphan penumbra of NOAA 11089 (area No. 2) over 25 h. In the continuum intensity maps, the penumbral filaments are observed to get shorter and the whole structure to decrease in size with time. In the LOS velocity maps, the patch with oppositely oriented flow disappears. As the orphan penumbra approaches the central meridian, the LOS velocities decrease. This is not immediately apparent because the maximum velocities exceed $5 \mathrm{~km} \mathrm{~s}^{-1}$ in all panels, but there is a decrease of $0.4 \mathrm{~km} \mathrm{~s}^{-1}$ of the median LOS velocity in the filaments between the first and last maps.

In the magnetic field panels of Fig. 10, one can see a shear movement of the opposite polarities. The negative polarity patch in the lower part moves westward (to the right) compared to the main positive polarity patch. Therefore, the filaments are oriented in the north/south direction in the first frame, while they are oriented in the north-east/south-west direction in the last frame. The upper segment of the orphan penumbra disappears, as the negative polarity patch originally located around $[10,20]$ arcsec disappears. This also happens later for the lower segment of the orphan penumbra when the negative polarity region disappears (see Sect. 3.3 below).

Additionally, we have tracked the evolution of the LOS magnetic field in NOAA 11089 for approximately $35 \mathrm{~h}$ using Hinode Na I D V/I magnetograms. From the centres of gravity of the sunspot umbrae, we find that the opposite-polarity leader and follower spots of NOAA 11089 separated with an average velocity of $130 \mathrm{~m} \mathrm{~s}^{-1}$, which is larger than the mean values found by Sobotka \& Roudier (2007, $80 \mathrm{~m} \mathrm{~s}^{-1}$ ) and Švanda et al. (2009, $45 \mathrm{~m} \mathrm{~s}^{-1}$ ). The leading negative polarity region was always highly structured, while the number density of magnetic features around the PIL decreased with time.

Figure 11 displays three co-spatial and co-temporal $G$-band images and NaI D V/I magnetograms summarizing the evolution of the orphan penumbra. It is clear that the penumbral filaments form between regions of opposite polarity, confirming the results presented in Sect. 3.1. The filaments are located in regions with weaker magnetogram signals than the surroundings. The differences between the Na I D V/I magnetograms of Fig. 11 and the magnetic field maps of Fig. 10 are caused mainly by the switch from the LOS frame to the LRF.

Played as a movie, the NaI D V/I magnetograms show the opposite-polarity regions approaching each other until the negative one disappears. There is a significant change in the size of the negative polarity patch, which is also clearly seen in Fig. 11. The positive polarity patches next to the orphan penumbra change their shapes with time but are preserved due to nearby pores that trail the leading sunspot. As there are no stable structures in the two regions, it is difficult to calculate their approach velocity. From the mean distance between the opposite-polarity patches (determined manually), we estimate the approach velocity to be around $40 \mathrm{~m} \mathrm{~s}^{-1}$. We obtained a similar velocity 

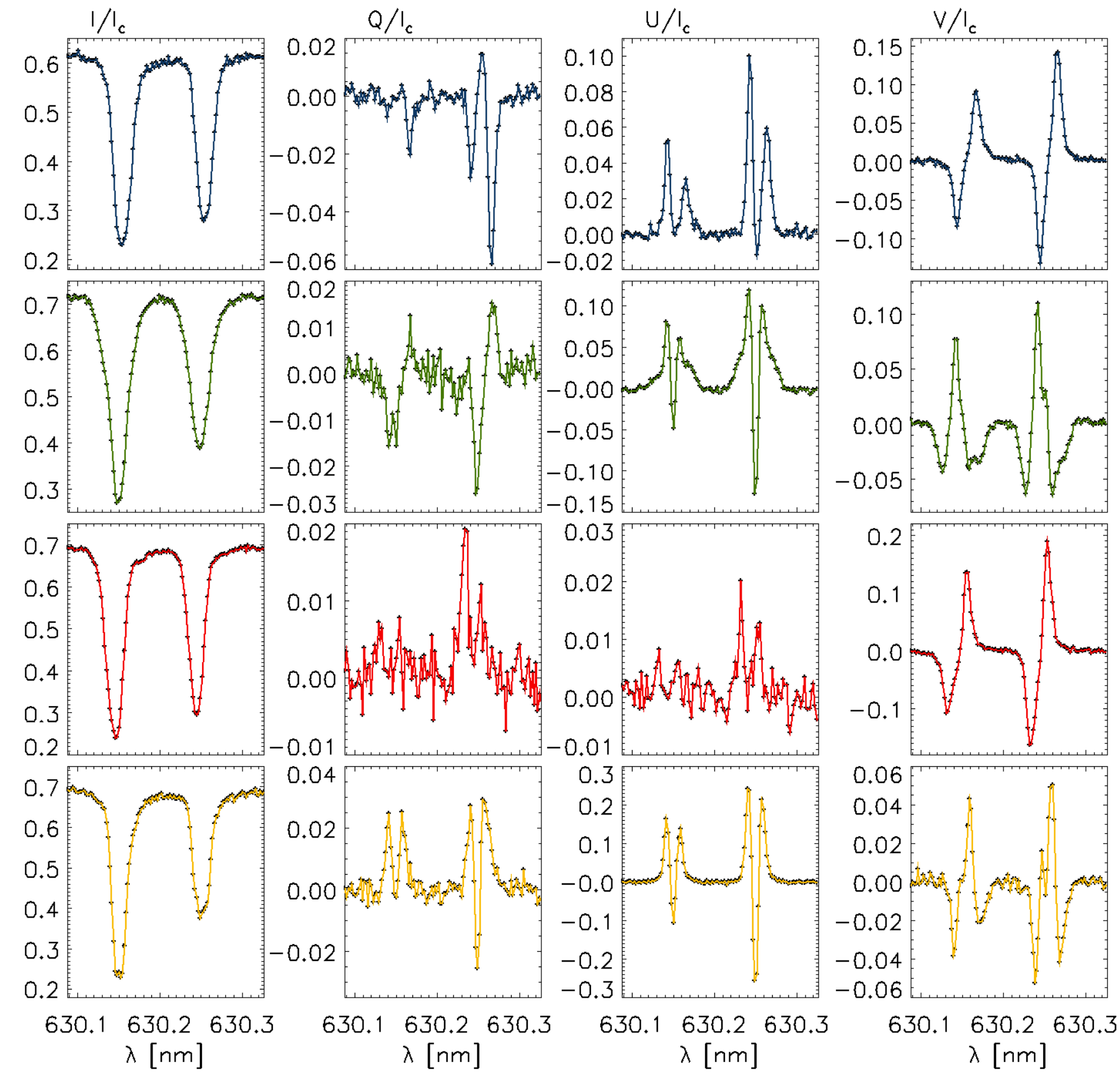

Fig. 9. Stokes profiles observed in the pixels marked with coloured + symbols in Fig. 8. From top to bottom, we show symmetric and asymmetric profiles in the orphan penumbra, along with symmetric and asymmetric profiles in the regular penumbra.

of $30 \mathrm{~m} \mathrm{~s}^{-1}$ by tracking the evolution of the length of the orphan penumbral filaments in the sequence of $G$-band images.

Since the two polarities approach with time and one polarity disappears according to the Na I D V/I magnetograms, the $\Omega$-shaped magnetic field responsible for the orphan penumbra must be submerging. The fragmented structure of the patches and their evolution also suggest that we do not observe a monolithic flux tube but a number of smaller tubes behaving in a similar way. There is also the possibility that the magnetic field in the negative polarity region dissipates, while it approaches the positive polarity region. However, we consider this a less plausible scenario because dissipating polarities in active regions tend to show increasing distances instead (van Driel-Gesztelyi \& Petrovay 1990; Moreno-Insertis et al. 1994).

\subsection{Brightness and apparent motions}

Figure 12 shows a late phase of the evolution of an orphan penumbra in the active region NOAA 10960. A sequence of images taken \pm 9 min around the $G$-band filtergram as displayed in Fig. $12 \mathrm{a}$, was used to derive apparent horizontal motions, $\boldsymbol{U}_{\mathrm{LCT}}$, in the low photosphere (Fig. 12b). Figure 12c shows the $\mathrm{Ca}$ II H filtergram co-spatial and co-temporal with Fig. 12a. Arrows in Fig. 12d represent the apparent motions in the upper photosphere derived from the $18 \mathrm{~min}$ sequence of $\mathrm{Ca}$ II $\mathrm{H}$ images. The arrows are shortened by a factor of two compared to Fig. 12b. In Table 1, we give the mean values of $\left|\boldsymbol{U}_{\mathrm{LCT}}\right|$ and their standard deviations in different parts of the $G$-band and Ca II H FOVs.

As can be seen in Fig. 12a, the morphology of orphan and regular penumbrae is very similar in the lower photosphere. Both 
J. Jurčák et al.: Orphan penumbrae: Submerging horizontal fields

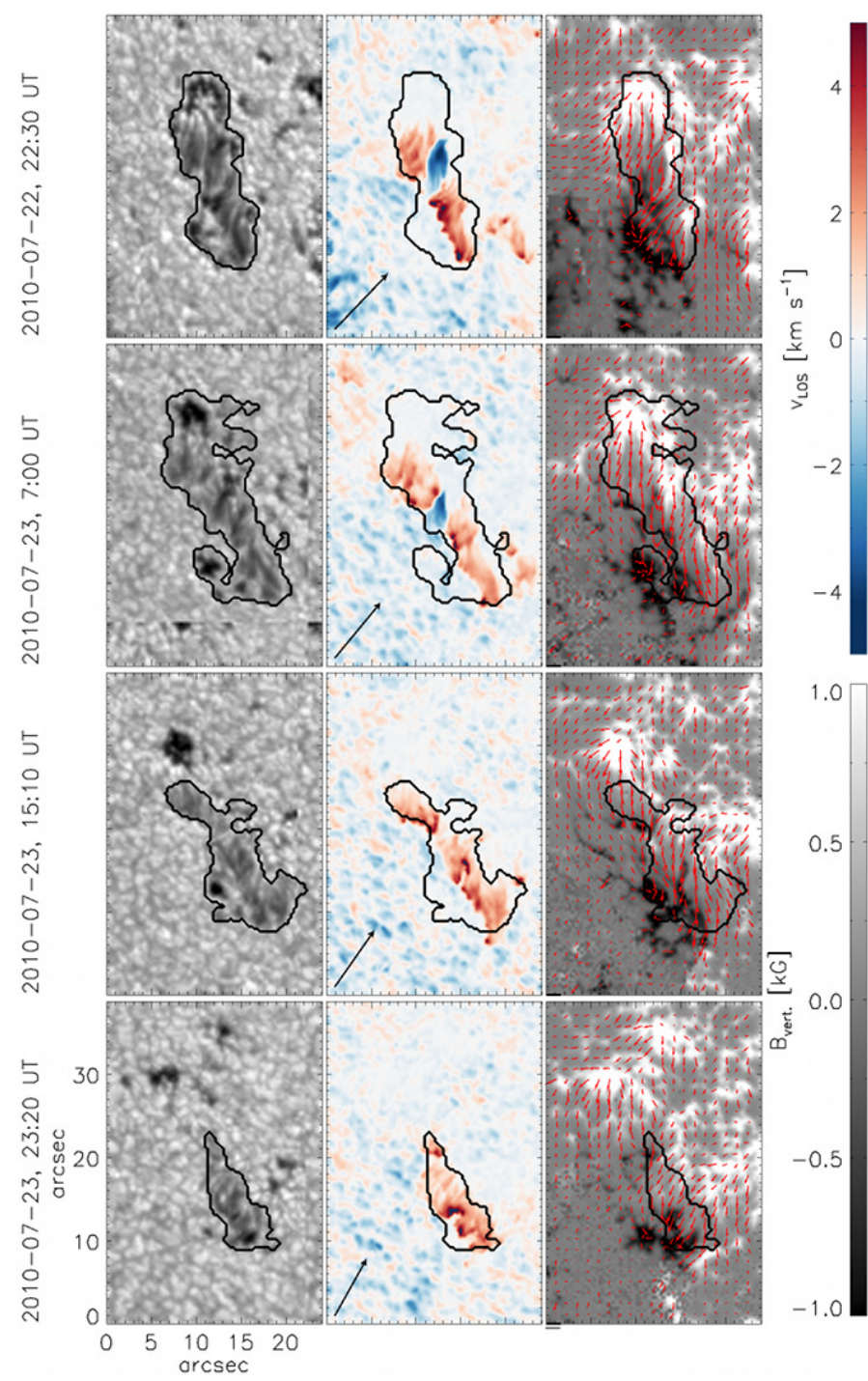

Fig. 10. Temporal evolution of the orphan penumbra observed in NOAA 11089 (area No. 2 in Fig. 3c). From left to right, we show continuum intensity maps, LOS velocity maps, and maps of the vertical component of the magnetic field with red arrows indicating the strength and orientation of the horizontal component. The black arrows in the LOS velocity maps point towards the disc centre.

have filaments with bright heads and darker tails. Figure 13a shows histograms of the $G$-band intensities in the two types of penumbrae. The shapes of the histograms are similar. The dark pixels with $I<0.5 I_{Q S}$ in the regular penumbra are a simple consequence of the umbra/penumbra boundary, which does not exist in the orphan penumbra. In both cases, the most frequent intensities are around $0.65 I_{Q S}$. The excess of pixels with intensities between $0.7 I_{Q S}<I<0.9 I_{Q S}$ in the orphan penumbra is partially caused by the bright regions near the outer boundary of the filamentary structure, which are proportionally larger compared to the regular penumbra. Even if these boundary regions are not taken into account, there is an excess of bright pixels in the orphan penumbra (not shown in Fig. 13a). On average, the orphan penumbra is slightly brighter than the regular penumbra when observed through the Hinode $G$-band filter.

In the $\mathrm{Ca}$ II $\mathrm{H}$ images, the orphan and regular penumbrae also have a similar appearance (Fig. 12c). However, a quantitative analysis reveals some differences. The histogram of $\mathrm{Ca}$ II $\mathrm{H}$ intensities in the regular penumbra has only one peak at $0.75 I_{Q S}$
Table 1. Apparent horizontal velocities.

\begin{tabular}{rcccc}
\hline \hline \multirow{2}{*}{ Region } & \multicolumn{2}{c}{$\overline{\left.\left|\boldsymbol{U}_{\mathrm{LCT} \mid}\right| \mathrm{km} \mathrm{s}^{-1}\right]}$} & \multicolumn{2}{|c}{$\sigma_{\left|U_{\mathrm{LCT}}\right|}\left[\mathrm{km} \mathrm{s}^{-1}\right]$} \\
& $G$-band & Ca II H & $G$-band & Ca II H \\
\hline Regular penumbra & 0.41 & 0.59 & 0.25 & 0.68 \\
inflow & 0.42 & 0.32 & 0.25 & 0.51 \\
outflow & 0.38 & 0.83 & 0.24 & 0.73 \\
Orphan penumbra & 0.45 & 0.86 & 0.28 & 0.55 \\
inflow & 0.29 & 0.18 & 0.15 & 0.10 \\
outflow & 0.53 & 0.89 & 0.29 & 0.55 \\
Moat region (RP) & 0.57 & 0.78 & 0.31 & 0.44 \\
Moat region (OP) & 0.54 & 0.69 & 0.23 & 0.44 \\
Quiet Sun & 0.57 & 0.86 & 0.31 & 0.46 \\
\hline
\end{tabular}

Notes. Mean values $\left(\overline{\left|\boldsymbol{U}_{\mathrm{LCT}}\right|}\right)$ and standard deviations $\left(\sigma_{\left|U_{\mathrm{LCT} \mid}\right|}\right)$ of apparent horizontal velocities in various regions of the solar atmosphere as determined from the $G$-band and $\mathrm{Ca}$ II $\mathrm{H}$ images. The abbreviations RP and OP stand for regular and orphan penumbra, respectively.

(Fig. 13c, solid line). For the orphan penumbra, the main peak is located at around $0.85 I_{Q S}$. There is a second peak around $1.0 I_{Q S}$, which is created by pixels near the orphan penumbra boundary. A hint of bright pixels near the outer edge of the regular penumbra can be seen as a bump of the solid line in Fig. 13c. Due to the different sizes of the two penumbrae, the boundary regions show up more prominently in the orphan penumbra histogram. Even if these regions are removed from the histograms, the $\mathrm{Ca}$ II $\mathrm{H}$ intensities of the filaments are not the same in the regular and orphan penumbrae (not shown in Fig. 13c). The orphan penumbra is brighter by approximately $0.1 I_{Q S}$, the intensity difference between the main peaks of Fig. 13c.

In Fig. 12b, we show the apparent flows found from the $G$-band filtergrams. The motion pattern in the regular penumbra confirms previous studies: in the inner penumbra, there are motions towards the sunspot umbra, while one observes outward motions in the outer penumbra (see Wang \& Zirin 1992; Sobotka et al. 1999; Márquez et al. 2006). Hereafter, motions opposite to the Evershed flow are referred to as inflows (inward motions) and flows in the direction of the Evershed flow as outflows (outward motions). The $\boldsymbol{U}_{\mathrm{LCT}}$ pattern in the orphan penumbra resembles that of the regular penumbra. Everywhere in the orphan penumbra, the apparent motions are aligned with the filaments. In regions corresponding to the inner penumbra, there are inward apparent motions (upper region of the orphan penumbra in Fig. 12). In regions corresponding to the outer penumbra, $\boldsymbol{U}_{\mathrm{LCT}}$ points outwards.

In Fig. 13b, we show the distribution of apparent horizontal motions as derived from the sequence of $G$-band images. The regular penumbra histogram made from all pixels (solid grey line) resembles the one found by Márquez et al. (2006) in a sunspot penumbra. The histogram peaks around $0.2 \mathrm{~km} \mathrm{~s}^{-1}$ with maximum values reaching $1.5 \mathrm{~km} \mathrm{~s}^{-1}$. The mean value of $\left|\boldsymbol{U}_{\mathrm{LCT}}\right|$ in the regular penumbra is $0.41 \mathrm{~km} \mathrm{~s}^{-1}$, which is about $0.1 \mathrm{~km} \mathrm{~s}^{-1}$ lower than the values obtained by Márquez et al. (2006) and Wang \& Zirin (1992). The distribution of apparent motions of all pixels in the orphan penumbra is shown by dashed grey line in Fig. 13b. Like in the regular penumbra, the most frequent velocities are around $0.3 \mathrm{~km} \mathrm{~s}^{-1}$ with a mean value of $0.45 \mathrm{~km} \mathrm{~s}^{-1}$. The main difference compared to the regular penumbra is the number of pixels with velocities around $1 \mathrm{~km} \mathrm{~s}^{-1}$. These flows are associated with outward motions (dashed black line - positive values) having a mean apparent velocity of $0.53 \mathrm{~km} \mathrm{~s}^{-1}$, which is faster than in the regular penumbra $\left(0.38 \mathrm{~km} \mathrm{~s}^{-1}\right.$, solid 


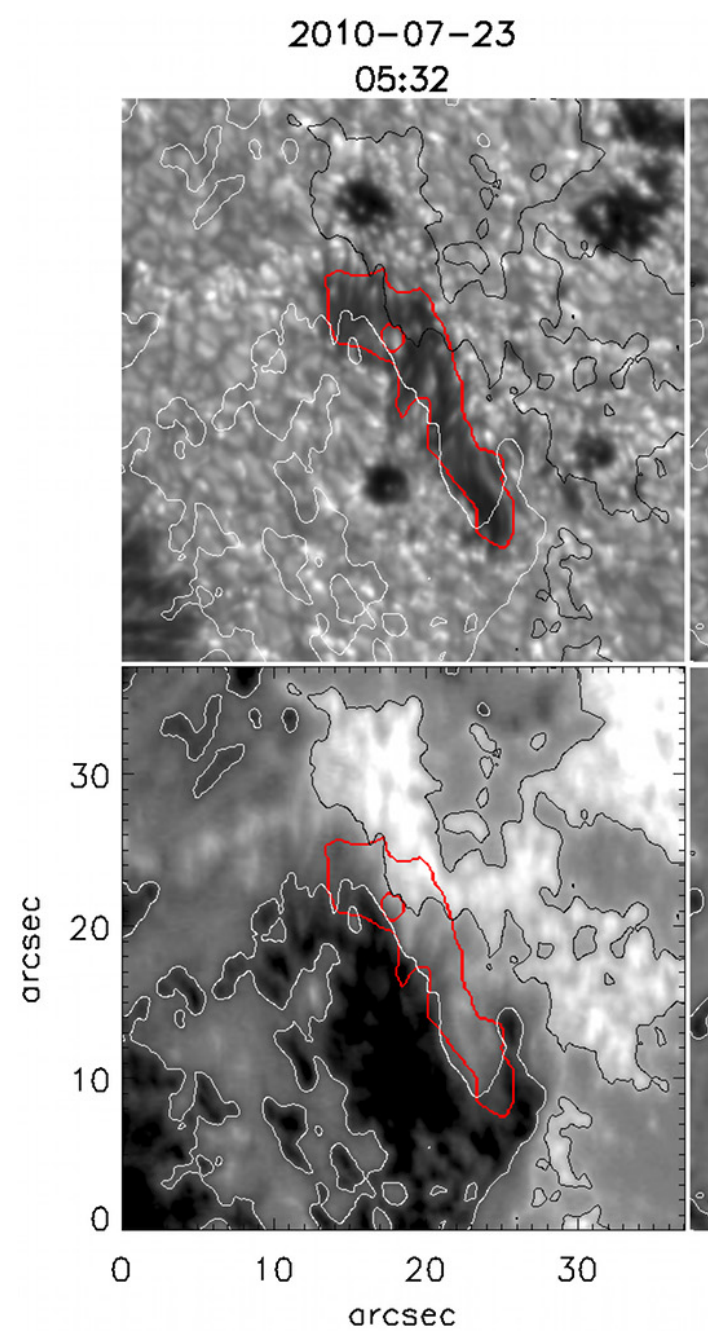

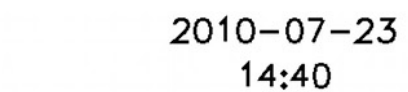
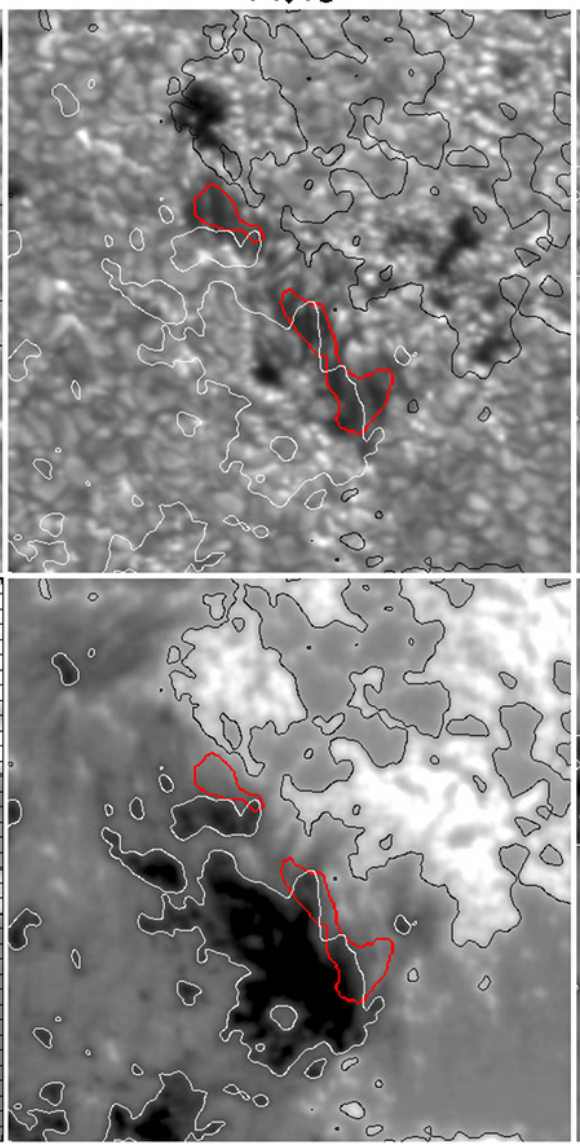

2010-07-24

$05: 39$
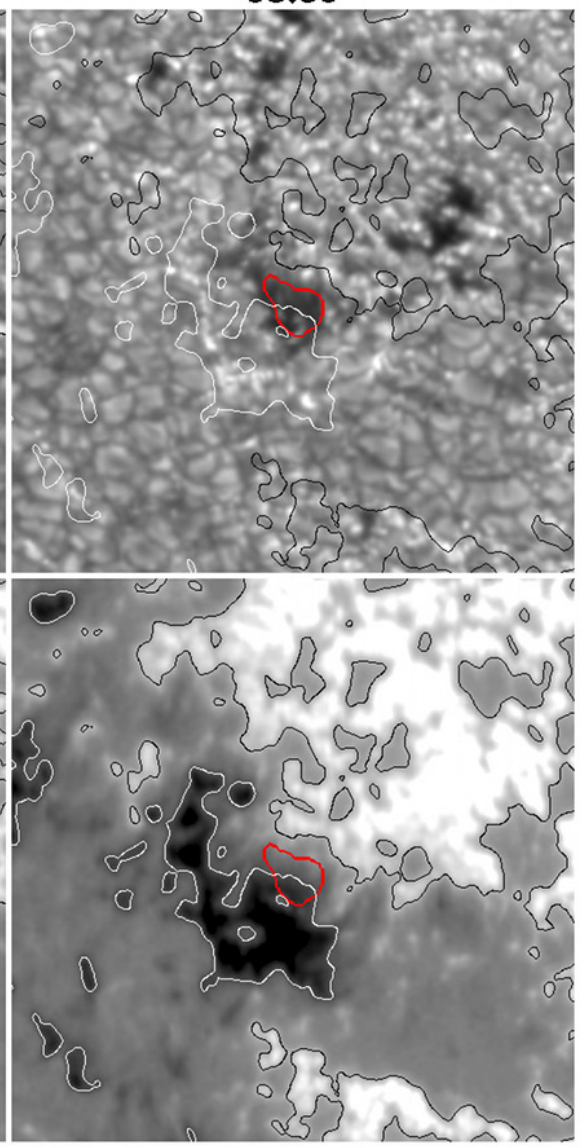

arcsec

Fig. 11. Co-spatial and co-temporal $G$-band images and NaI D V/I magnetograms showing the temporal evolution of the orphan penumbra observed in active region NOAA 11089 (area No. 2 in Fig. 3c). The red contours mark the orphan penumbra location. The black and white contours show the patches of positive and negative polarity, respectively. The temporal evolution of the V/I magnetograms (lower panels) is available in the online edition. (Online movie)

black lines - positive values). On the other hand, the mean inward $\boldsymbol{U}_{\mathrm{LCT}}$ is $0.29 \mathrm{~km} \mathrm{~s}^{-1}$ (dashed black line - negative values), which is slower than in the regular penumbra $\left(0.42 \mathrm{~km} \mathrm{~s}^{-1}\right.$, solid black lines - negative values, see also Table 1). This might be partially explained by the position of the contour around the orphan penumbra, which does not encircle most of the bright penumbral grains whose motions give rise to inward flows.

The apparent motions derived from the Ca II $\mathrm{H}$ filtergrams are presented in Fig. 12d. The pattern in the regular penumbra is the same as in Fig. 12b, which shows inward motions in the inner penumbra and outward motions in the outer penumbra. However, there is an important difference between the areas occupied by inward and outward motions in the $G$-band and Ca II H maps. The yellow dashed contours in Figs. 12a,c mark the boundary between oppositely oriented flows. This boundary moves significantly towards the sunspot umbra in the Ca II $\mathrm{H}$ map. The inward flows are determined by the LCT method from the apparent motions of bright penumbral grains. As these structures are confined to the low photosphere (Jurčák et al. 2007; Sobotka \& Jurčák 2009), they are prominent in the $G$-band filtergrams but not in the $\mathrm{Ca}$ II $\mathrm{H}$ images. Therefore, the inward motions are weaker and occupy smaller areas in Ca II H. The motion patterns of the orphan penumbra are also similar in the upper and lower photosphere (Figs. 12d and 12b). The inward motions almost completely disappear in the orphan penumbra for the same reason as in the regular penumbra. This also occurs because the orphan penumbra boundary (red contour in Figs. 12a,c) does not include the area where the bright penumbral grains are most common.

The distributions of apparent motions derived from the sequence of Ca II $\mathrm{H}$ images are, however, significantly different in regular and orphan penumbrae, as can be seen in Fig. 13d. In the regular penumbra (solid grey line), there is a main peak at $0.2 \mathrm{~km} \mathrm{~s}^{-1}$, which is mostly created by inward motions (with mean $\boldsymbol{U}_{\mathrm{LCT}}$ of $0.32 \mathrm{~km} \mathrm{~s}^{-1}$, solid black line - negative values). The outward flows have a flat distribution with mean velocities of $0.83 \mathrm{~km} \mathrm{~s}^{-1}$ and maximum values of $2 \mathrm{~km} \mathrm{~s}^{-1}$ (solid black line - positive values). In the orphan penumbra, there are almost no regions with inflows (The mean value of the inflow $\boldsymbol{U}_{\mathrm{LCT}}$ is $0.18 \mathrm{~km} \mathrm{~s}^{-1}$ with a maximum values of $0.4 \mathrm{~km} \mathrm{~s}^{-1}$, which is shown as a dashed black line - negative values.). The distribution of outflows is similar in the orphan and the regular penumbra, which is a flat distribution with maximum velocities over $2 \mathrm{~km} \mathrm{~s}^{-1}$ and mean flows of $0.89 \mathrm{~km} \mathrm{~s}^{-1}$ (dashed black line - positive values). Taking into account all the pixels in the regular and orphan penumbrae, the mean apparent motions are $0.59 \mathrm{~km} \mathrm{~s}^{-1}$ 

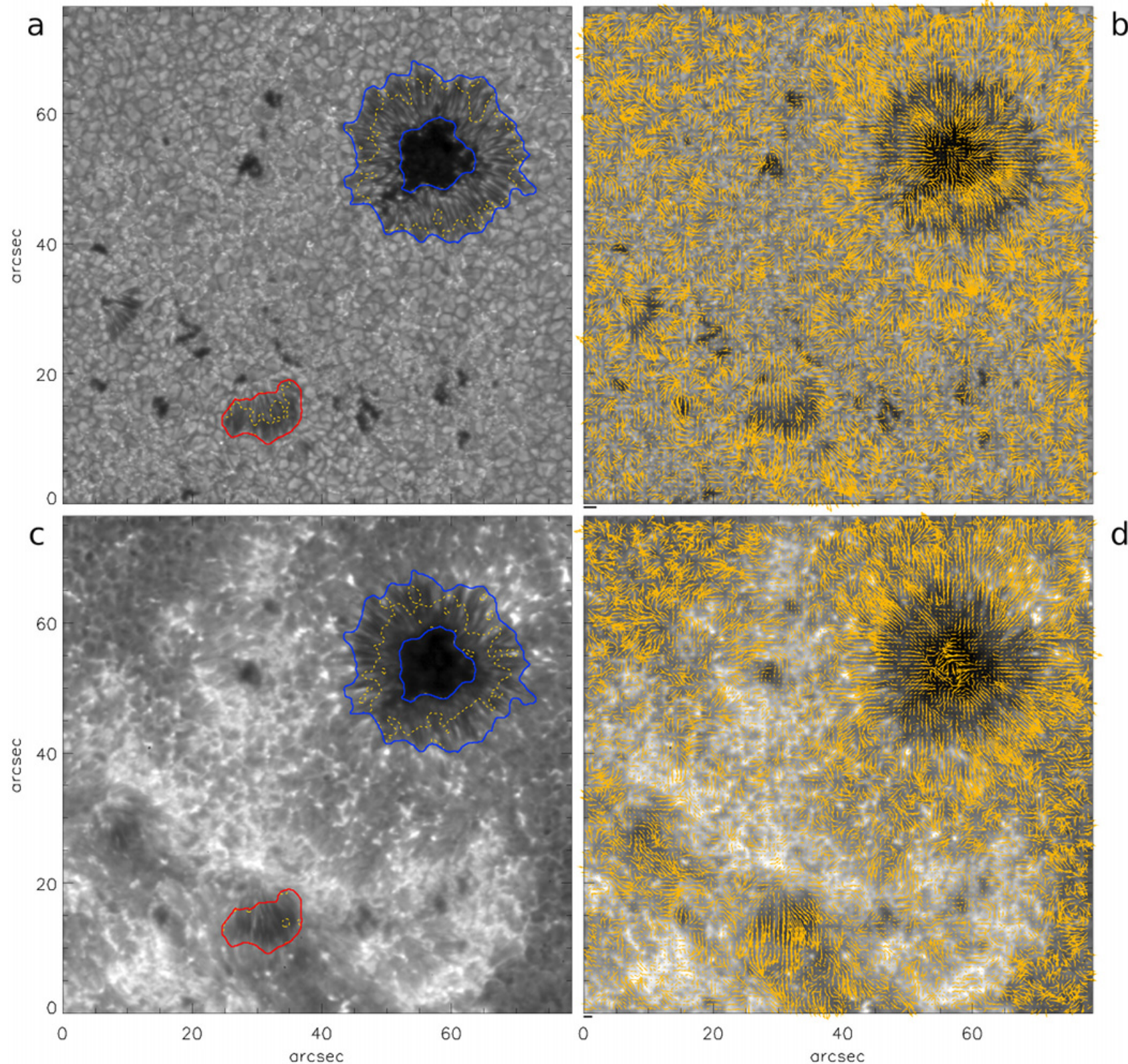

Fig. 12. $G$-band and CaII H filtergrams of active region NOAA 10960 taken at 15:51 UT on 6 June 2007 a), c). The red and blue contours mark the position of orphan and regular penumbrae, respectively, and are based on smoothed $G$-band intensity images. The arrows show the apparent horizontal motions of structures in the $G$-band $\mathbf{b}$ ) and Ca II $\mathrm{H} \mathrm{d}$ ) filtergrams. The thick lines below the beginning of the $x$-axes correspond to $1 \mathrm{~km} \mathrm{~s}^{-1}$. The yellow dashed contours in a) and c) mark the boundary between the inward and outward apparent motions seen in b) and d), respectively.

and $0.86 \mathrm{~km} \mathrm{~s}^{-1}$, respectively (see Table 1 ). The flows are faster compared to the lower atmospheric layers due to the significant increase of apparent velocity of the outward flows.

In both the $G$-band and $\mathrm{Ca}$ II $\mathrm{H}$ horizontal flow maps, one can distinguish a moat-flow region around the main sunspot except for the lower left area, where the penumbra is not fully developed. This agrees with recent studies of the moat flow (Vargas Domínguez et al. 2007, 2008). The apparent motions in the orphan penumbra also extend their boundaries in the direction of the flow, creating a structure comparable to a sunspot moat region. In the deep photosphere, the apparent motions exhibited by the orphan penumbra "moat" region have amplitudes comparable to the outward motions found inside the penumbra, while the moat flows around the sunspot have higher velocities than their penumbral counterparts (see Table 1). In the upper photosphere, the velocities of the apparent motions detected in the moat regions are smaller than those found in the penumbra, although the difference is small in the case of the regular penumbra. The absolute values of the moat $\boldsymbol{U}_{\mathrm{LCT}}$ increase as one moves higher in the atmosphere (see Table 1 and also Sobotka \& Roudier 2007).

\section{Discussion}

As shown in Sect. 3, there are many similarities between regular and orphan penumbrae. Their filaments look identical in the atmospheric layers probed by the $G$-band and $\mathrm{Ca}$ II $\mathrm{H}$ images. In the deep photosphere, orphan penumbrae are only slightly brighter than regular penumbrae, while the intensity difference is significant in the upper photosphere, as demonstrated by 

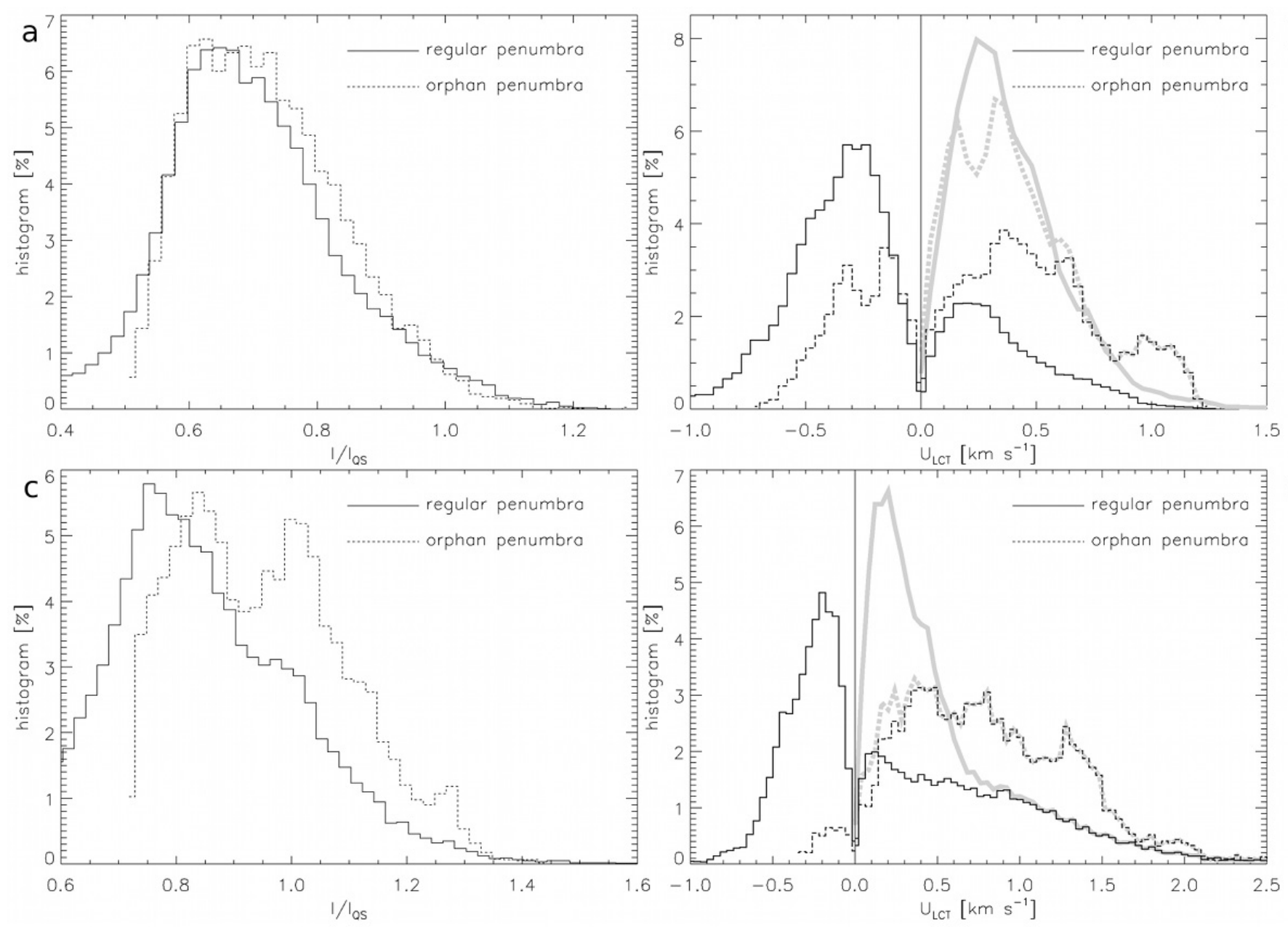

Fig. 13. a) and b) Histograms of the $G$-band intensity and apparent horizontal velocities of pixels encircled by contours in Fig. 12a. c) and d) Histograms of the $\mathrm{Ca}$ II $\mathrm{H}$ intensity and apparent horizontal velocities of pixels encircled by contours in Fig. 12c. Inward motions have negative sign. The thick grey lines in the histograms of apparent horizontal velocities show the distribution of $\left|\boldsymbol{U}_{\mathrm{LCT}}\right|$.

the Ca II H filtergrams. Such an intensity difference could be explained by the filaments reaching different heights in orphan and regular penumbrae. The background component of the magnetic field in the latter creates a magnetic canopy (Borrero et al. 2008) that may limit the geometrical height attainable by the flow channels containing the hot gas, whereas the former would not be impeded in any way.

Despite the possible height difference, the filaments of orphan and regular penumbrae share a common feature: their heads (bright penumbral grains) and tails are low-lying structures visible only in the $G$-band filtergrams. When we compare the $\mathrm{Ca}$ II $\mathrm{H}$ with the $G$-band images, the filaments appear shorter in the upper photosphere and do not reach the boundaries based on $G$-band intensities (Figs. 12a,c). This agrees with the magnetic configuration we have deduced for the orphan penumbrae, where the magnetic field is surfacing and submerging at the heads and tails of the filaments. The shortest filaments on the edges of orphan penumbrae cannot be seen in the $\mathrm{Ca}$ II $\mathrm{H}$ filtergrams. There is also a small segment of orphan penumbra located around $[10,30]$ arcsec in Figs. 12a,c, which is obviously smaller in the $\mathrm{Ca}$ II $\mathrm{H}$ image compared to the $G$-band filtergram.

The general properties of the observed apparent horizontal motions are similar in orphan and regular penumbrae, which are inward motions near the filament heads and outward motions around the tails. The shapes of the histograms of inflows and outflows are also comparable. As shown by Vargas Domínguez et al. (2008), the moat flow seems to be closely related to the presence of the Evershed flow. Therefore, it is interesting to compare the mean outflow velocities found in orphan and regular penumbrae with the flows observed in their moat regions (see Table 1). The moat flows are faster around the regular penumbra in the two photospheric layers probed by the observations, although the outward motions are faster in orphan penumbrae and we also find faster "Evershed" flows there. Such a discrepancy may support the results of Löhner-Böttcher \& Schlichenmaier (2013), who found no correlation between the Evershed and moat flow velocities and interpreted this as a hint that the two flows have different physical origins. However, the presence of moat flows on the sides of orphan penumbrae towards which the apparent motions are directed supports the conclusions of Vargas Domínguez et al. (2008).

We cannot directly confirm the horizontal direction of the gas flow in orphan penumbrae. However, the LOS velocity measured in the orphan and regular penumbrae of active regions NOAA 10960 and 11089 show the same decreasing trend as they approach the central meridian. Some orphan penumbral filaments are also almost perpendicular to the direction towards disc centre and exhibit very small LOS velocities. Since the magnetic field in the filaments is mostly horizontal, it is reasonable to assume that the flow is significantly inclined. Observations of 


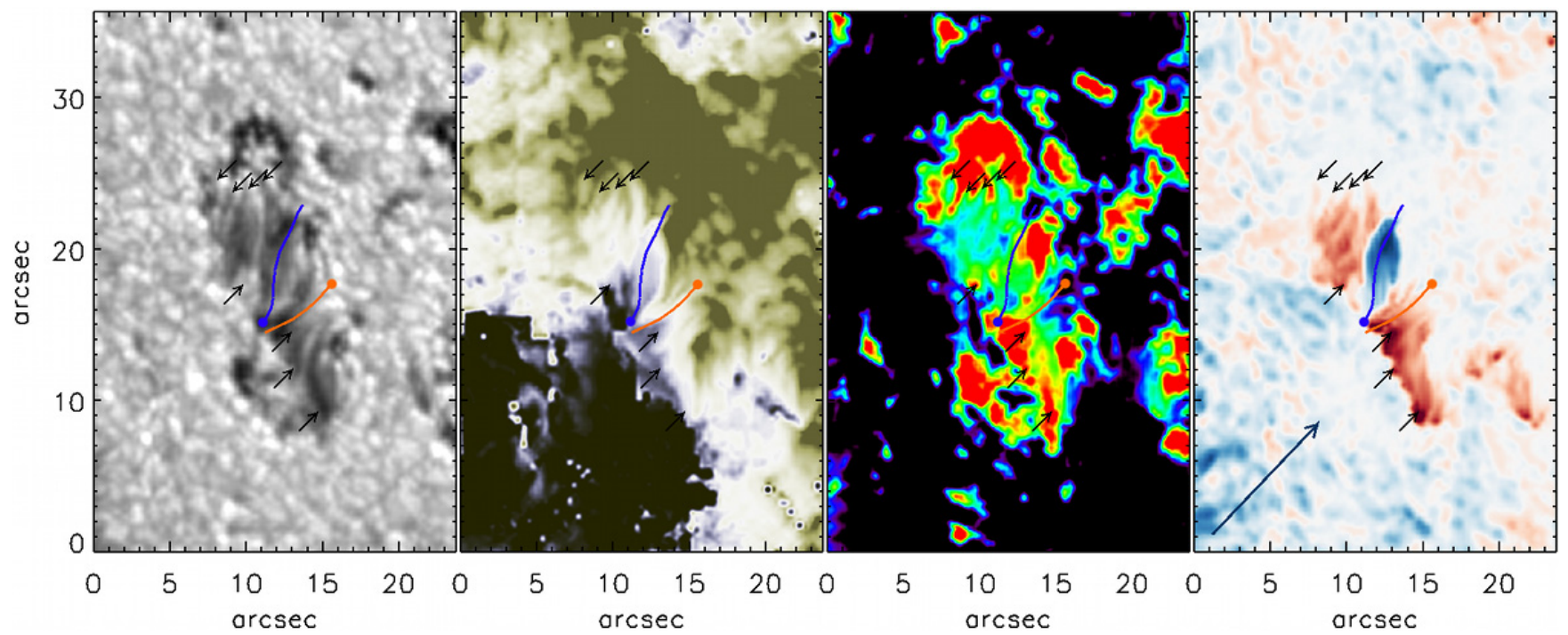

Fig. 14. Examples of orphan penumbral filaments in area No. 2. The panels are identical here and in Fig. 5. The small arrows pointing up and down (in the lower and upper parts of the maps) mark the tails and heads of selected filaments, respectively. The blue and orange lines follow filaments with oppositely oriented flows. The large arrow points toward the disc centre.

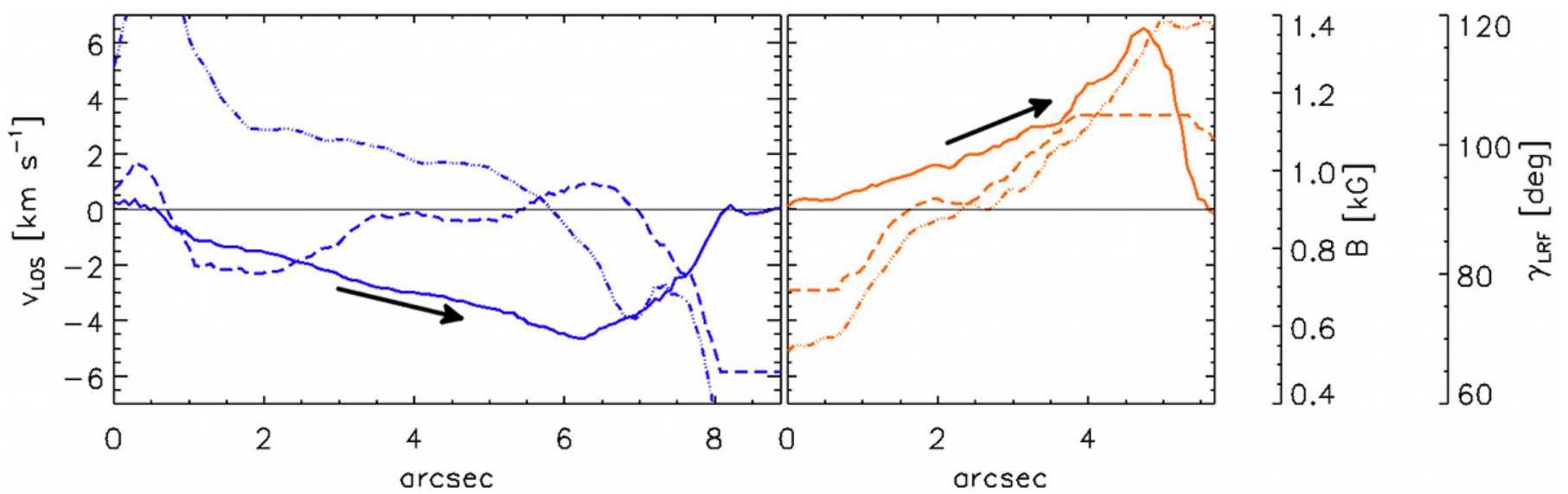

Fig. 15. Variation of magnetic field strength (dashed lines), LRF inclination (dash-dotted lines), and LOS velocity (solid lines) along the filaments marked by orange and blue lines in Fig. 14. Distances are measured from the filament heads (circles in Fig. 14). The black arrows show the direction of the flows along the cuts.

NOAA 10960 taken close to the disc centre show fast downflows at the end of orphan penumbral filaments, which are analogous to the downflows observed in the outer parts of regular penumbrae. There is no clear evidence of upflows in bright grains of the orphan penumbra, but these are also not detectable in the nearby regular penumbra (possibly due to insufficient spatial resolution).

To understand the origin of the gas flows, we need to examine how they change along penumbral filaments. The arrows pointing down in Fig. 14 mark the heads of a few selected filaments, where the flow appears (rightmost panel). These regions are co-spatial with spines that have weaker and more horizontal magnetic field (middle panels) and with bright penumbral grains (leftmost panel). There is a slight offset between the arrow heads and the bright grains in the continuum intensity map. As mentioned in Sect. 2, the maps of physical parameters correspond to a geometrical height around $150 \mathrm{~km}$, while the continuum intensity is formed around $0 \mathrm{~km}$. This height difference with the location approximately $40^{\circ}$ from the disc centre can explain the observed offset. The arrows pointing up mark the regions, where the filaments submerge below the solar surface. These areas are associated with stronger flows (left map) and correspond to regions with locally stronger magnetic fields pointing towards the solar surface (middle maps).

The blue and orange lines in Fig. 14 mark two individual filaments with oppositely oriented flows. They have been tracked manually using the continuum intensity, LOS velocity, and magnetic field inclination of the structures. Figure 15 shows the LOS velocity $\left(v_{\text {LOS }}\right)$, magnetic field strength $(B)$, and LRF inclination $\left(\gamma_{\mathrm{LRF}}\right)$ along these paths. The blue lines correspond to the cut through the filament with blueshifts. The flow forms in regions with $B$ around $750 \mathrm{G}$ and $\gamma_{\mathrm{LRF}}$ around $110^{\circ}$ (negative polarity patch). There is a gradual increase of the LOS velocity up to $-5 \mathrm{~km} \mathrm{~s}^{-1}$, where the magnetic field strength reaches $950 \mathrm{G}$ and turns towards the solar surface with an inclination of around $80^{\circ}$ (positive polarity patch). In the filament with redshifts, we also find a flow towards a stronger magnetic field. The flow forms in regions with $B$ of $650 \mathrm{G}$, while the maximum value of $1400 \mathrm{G}$ is reached at the location with the highest LOS velocity of $6.5 \mathrm{~km} \mathrm{~s}^{-1}$. The filament shown in red starts in a positive 
polarity patch $\left(\gamma_{\mathrm{LRF}}=70^{\circ}\right)$ and submerges in a negative patch $\left(\gamma_{\mathrm{LRF}}=120^{\circ}\right)$. This configuration of the flow and the magnetic field clearly indicates a siphon flow.

The siphon flow mechanism was suggested as a possible driver of the Evershed flow by Meyer \& Schmidt (1968): the difference of magnetic field strength at the footpoints of a loop results in different gas pressures, which generates a mass flow towards the footpoint with stronger magnetic field (Thomas 1988; Degenhardt 1991). This seemed to be an unrealistic scenario for sunspot penumbrae because most analyses showed significantly weaker magnetic fields in the outer penumbra. Some studies suggest that the field can be stronger than the background field component (Bellot Rubio et al. 2004; Tritschler et al. 2007; Ichimoto et al. 2008; Borrero \& Solanki 2008), and there are analyses that support the siphon flow mechanism indirectly (Borrero et al. 2005; Montesinos \& Thomas 1997). More recent studies using complex inversion schemes found strong magnetic fields co-spatial with submerging Evershed flow channels in sunspot penumbrae (Tiwari et al. 2013; van Noort et al. 2013). Rempel (2011) made the suggestion that the driver of the flow could be the strong horizontal component of the Lorentz force. He also stated that siphon-like flow channels can be produced by such a process in the outer penumbra but that they are more a consequence of the fast outflow than its cause. Our analysis does not allow us to decide if the magnetic configuration we have found in orphan penumbrae is the source or the consequence of the fast flow.

Taking into account all the similarities between orphan and regular penumbrae, we propose that orphan penumbral filaments represent the flow channels of regular penumbrae, which are not surrounded or confined by a stronger and more vertical background field. This is also supported by the studies of Lim et al. (2013) and Zuccarello et al. (2014), who found no changes in the structure of chromospheric magnetic fields during the formation of orphan penumbrae.

The magnetic configuration we have deduced for orphan penumbrae is that of a flat $\Omega$-loop. The temporal evolution of the opposite-polarity regions surrounding them suggests that the disappearance of orphan penumbrae is caused by the submergence of the $\Omega$-loop. However, this process is very slow, and we do not detect the corresponding Doppler shifts. Even the observations of NOAA 10960 at an heliocentric angle of $8^{\circ}$ did not reveal downflows in the horizontal part of the filaments. Assuming that the height of the structure creating the orphan penumbra is comparable to its width, a disappearance time of $30 \mathrm{~h}$ would result in a submergence velocity of $40 \mathrm{~m} \mathrm{~s}^{-1}$. Due to the horizontal gas flows, the local variations of the LOS velocity observed within the orphan penumbrae are much larger than this, making it impossible to detect the small downward motion of the loop as a whole.

Our interpretation that the disappearance of orphan penumbrae is the consequence of the submergence of an $\Omega$-shaped magnetic loop does not contradict with Lim et al. (2013) and Zuccarello et al. (2014). They find that orphan penumbrae are created by emerging $\Omega$-loops that are trapped in the photosphere by an overlaying chromospheric magnetic field but did not discuss the cause of orphan penumbral disappearance in detail.

\section{Conclusions}

We thoroughly investigated orphan penumbrae, which are filamentary structures resembling sunspot penumbrae but are not connected to an umbra that lie near PILs in active regions. We observed these structures in two active regions and used Hinode SOT data to analyse their properties.

In the $G$-band, orphan and regular penumbrae have the same morphology, although the former are slightly brighter on average. The intensity difference is more pronounced in the upper photosphere, which is probed by the $\mathrm{Ca}$ II $\mathrm{H}$ filtergrams (Figs. 13a,c). We suspect the reason for the enhanced intensity of the filaments in orphan penumbrae is their more elevated geometrical height, as they are not limited by the magnetic canopy of the background field present in sunspots.

Although there are some differences in the amplitudes of apparent motions in orphan and regular penumbrae, their overall structure is the same in the low and upper photosphere. We observe apparent motions opposite to the gas flow in the inner penumbra (heads of the filaments) and apparent motions following the flow in the outer parts of orphan and regular penumbrae (Figs. 13b 13d). Similarly to sunspots, there is a moat region near orphan penumbrae, which is located in the area towards which the flow is directed (extending the relation between the Eveshed flow and moat region found by Vargas Domínguez et al. 2007 to orphan penumbrae).

The analysis of Hinode SP and NFI data shows that orphan penumbrae are magnetic structures with the shape of flat $\Omega$ loops. During the evolution of orphan penumbrae, the opposite polarities flanking them approach each other, while the filaments get shorter and disappear, which points to the submergence of the whole structure. Our velocity measurements cannot be used to demonstrate the downward motion as they are dominated by "Evershed" flows, and even observations taken close to the disc centre do not exhibit downward motions. We also cannot distinguish whether the filaments disappear earlier in the Ca II H images or the $G$-band filtergrams.

The LOS velocities observed in orphan penumbrae are larger than those of nearby regular penumbrae, but the structure of the flow is the same. The lowest velocities occur near the filament heads, while the flow speed increases along the filaments and reaches a maximum in the tails. Using full Stokes polarimetry, we were able to determine the orientation and strength of the magnetic field along individual filaments of orphan penumbrae (Figs. 5, 10, 14, and 15). We found that the flows are oriented towards the footpoints with a stronger magnetic field, which agrees with the siphon flow mechanism as the driver of the flow.

Due to the similarities between orphan and regular penumbrae, we tentatively propose that orphan penumbrae represent the weaker and more inclined magnetic field component of regular penumbrae (not influenced by the background field present in sunspots, Solanki \& Montavon 1993). On the other hand, this paper supports the siphon flow mechanism as the driver of the Evershed flow. It remains to be studied if the observed magnetic field configuration is just a consequence of the fast flow (as suggested by Rempel 2011) or the actual driver of the flow.

Acknowledgements. The support from GA CR P209/12/0287 and RVO:67985815 is gratefully acknowledged. This work has been funded by the Spanish MINECO through project AYA2012-39636-C06-05, including a percentage from European FEDER funds. Hinode is a Japanese mission developed and launched by ISAS/JAXA, with NAOJ as domestic partner and NASA and STFC (UK) as international partners. It is operated by these agencies in cooperation with ESA and NSC (Norway).

\section{References}

Bellot Rubio, L. R., Balthasar, H., \& Collados, M. 2004, A\&A, 427, 319 Borrero, J. M., \& Ichimoto, K. 2011, Liv. Rev. Sol. Phys., 8, 4 Borrero, J. M., \& Solanki, S. K. 2008, ApJ, 687, 668 
Borrero, J. M., Solanki, S. K., Bellot Rubio, L. R., Lagg, A., \& Mathew, S. K. 2004, A\&A, 422, 1093

Borrero, J. M., Lagg, A., Solanki, S. K., \& Collados, M. 2005, A\&A, 436, 333

Borrero, J. M., Lites, B. W., \& Solanki, S. K. 2008, A\&A, 481, L13

Brants, J. J., \& Steenbeek, J. C. M. 1985, Sol. Phys., 96, 229

Cabrera Solana, D., Bellot Rubio, L. R., \& del Toro Iniesta, J. C. 2005, A\&A, 439, 687

Carlsson, M., Hansteen, V. H., de Pontieu, B., et al. 2007, PASJ, 59, 663

Chandrasekhar, S. 1961, Hydrodynamic and hydromagnetic stability (Oxford: Clarendon Press)

Cheung, M. C. M., Schüssler, M., \& Moreno-Insertis, F. 2007, A\&A, 461, 1163 Degenhardt, D. 1991, A\&A, 248, 637

Ichimoto, K., Tsuneta, S., Suematsu, Y., et al. 2008, A\&A, 481, L9

Ishikawa, R., Tsuneta, S., \& Jurčák, J. 2010, ApJ, 713, 1310

Jess, D. B., Shelyag, S., Mathioudakis, M., et al. 2012, ApJ, 746, 183

Jurčák, J. 2011, A\&A, 531, A118

Jurčák, J., Bellot Rubio, L., Ichimoto, K., et al. 2007, PASJ, 59, 601

Keppens, R., \& Martinez Pillet, V. 1996, A\&A, 316, 229

Kosugi, T., Matsuzaki, K., Sakao, T., et al. 2007, Sol. Phys., 243, 3

Kuckein, C., Martínez Pillet, V., \& Centeno, R. 2012, A\&A, 539, A131

Leenaarts, J., Rutten, R. J., Reardon, K., Carlsson, M., \& Hansteen, V. 2010, ApJ, 709, A1362

Leka, K. D., Barnes, G., \& Crouch, A. 2009, in The Second Hinode Science Meeting: Beyond Discovery-Toward Understanding, eds. B. Lites, M. Cheung, T. Magara, J. Mariska, \& K. Reeves, ASP Conf. Ser., 415, 365

Lim, E.-K., Yurchyshyn, V., Goode, P., \& Cho, K.-S. 2013, ApJ, 769, L18

Lites, B. W., Low, B. C., Martínez Pillet, V., et al. 1995, ApJ, 446, 877

Löhner-Böttcher, J., \& Schlichenmaier, R. 2013, A\&A, 551, A105

Loughhead, R. E., \& Bray, R. J. 1961, Austral. J. Phys., 14, 347

Márquez, I., Sánchez Almeida, J., \& Bonet, J. A. 2006, ApJ, 638, 553

Metcalf, T. R. 1994, Sol. Phys., 155, 235

Meyer, F., \& Schmidt, H. U. 1968, Z. Ang. Math. Mech., 48, T218

Miller, R. 1960, J. British Astron. Assoc., 70, 100

Montesinos, B., \& Thomas, J. H. 1997, Nature, 390, 485

Moreno-Insertis, F., Caligari, P., \& Schuessler, M. 1994, Sol. Phys., 153, 449
November, L. J., \& Simon, G. W. 1988, ApJ, 333, 427

Rempel, M. 2011, ApJ, 729, 5

Rezaei, R., Bruls, J. H. M. J., Schmidt, W., et al. 2008, A\&A, 484, 503

Ruiz Cobo, B., \& del Toro Iniesta, J. C. 1992, ApJ, 398, 375

Sainz Dalda, A., Martínez-Sykora, J., Bellot Rubio, L., \& Title, A. 2012, ApJ, 748,38

Schlichenmaier, R. 2009, Space Sci. Rev., 144, 213

Schlichenmaier, R., Rezaei, R., Bello González, N., \& Waldmann, T. A. 2010, A\&A, 512, L1

Shelyag, S., Schüssler, M., Solanki, S. K., Berdyugina, S. V., \& Vögler, A. 2004, A\&A, 427, 335

Sobotka, M., \& Jurčák, J. 2009, ApJ, 694, 1080

Sobotka, M., \& Roudier, T. 2007, A\&A, 472, 277

Sobotka, M., Brandt, P. N., \& Simon, G. W. 1999, A\&A, 348, 621

Solanki, S. K. 2003, A\&ARv, 11, 153

Solanki, S. K., \& Montavon, C. A. P. 1993, A\&A, 275, 283

Tarbell, T., Ferguson, S., Frank, Z., et al. 1990, in Solar Photosphere: Structure, Convection, and Magnetic Fields, ed. J. O. Stenflo, IAU Symp., 138, 147

Thomas, J. H. 1988, ApJ, 333, 407

Thomas, J. H., \& Weiss, N. O. 2008, Sunspots and Starspots (Cambridge University Press)

Tiwari, S. K., van Noort, M., Lagg, A., \& Solanki, S. K. 2013, A\&A, 557, A25

Tritschler, A., Müller, D. A. N., Schlichenmaier, R., \& Hagenaar, H. J. 2007, ApJ, 671, L85

Tsuneta, S., Ichimoto, K., Katsukawa, Y., et al. 2008, Sol. Phys., 249, 167

Švanda, M., Klvaňa, M., \& Sobotka, M. 2009, A\&A, 506, 875

van Driel-Gesztelyi, L., \& Petrovay, K. 1990, Sol. Phys., 126, 285

van Noort, M., Lagg, A., Tiwari, S. K., \& Solanki, S. K. 2013, A\&A, 557, A24

Vargas Domínguez, S., Bonet, J. A., Martínez Pillet, V., et al. 2007, ApJ, 660, L165

Vargas Domínguez, S., Rouppe van der Voort, L., Bonet, J. A., et al. 2008, ApJ, 679,900

Viticchié, B. 2012, ApJ, 747, L36

Wang, H., \& Zirin, H. 1992, Sol. Phys., 140, 41

Zirin, H., \& Wang, H. 1991, Adv. Space Res., 11, 225

Zuccarello, F., Guglielmino, S. L., \& Romano, P. 2014, ApJ, submitted 